\title{
1 A Stochastic Model for Particulate Suspension 2 Flow in Porous Media
}

\section{A. SANTOS and P. BEDRIKOVETSKY*}

4 North Fluminense State University (LENEP/UENF), Brazil

5 (Received: 3 January 2003; accepted in final form: 3 April 2005)

6 Abstract. A population balance model for a particulate suspension transport with size 7 exclusion capture of particles by porous rock is derived. The model accounts for particle 8 flux reduction and pore space accessibility due to restriction for large particles to move

9 through smaller pores - a particle is captured by a smaller pore and passes through a 10 larger pore. Analytical solutions are obtained for a uniform pore size medium, and also 11 for a medium with small pore size variation. For both cases, the equations for averaged 12 concentrations significantly differ from the classical deep bed filtration model.

13 Key words: deep bed filtration, pore size exclusion, accessibility, stochastic model, averaging.

\section{Nomenclature}

$c \quad$ total suspended particle concentration, $\mathrm{L}^{-3}$.

$C \quad$ concentration distribution for suspended particles, $\mathrm{L}^{-4}$.

$f \quad$ size distribution (probability distribution function), $\mathrm{L}^{-1}$.

$\frac{f_{T}}{h} \quad$ size distribution of $r_{\mathrm{s}}$-particle population retained in $r_{\mathrm{p}}$-pores, $\mathrm{L}^{-2}$.

$\frac{f_{T}}{h} \quad$ total vacant pore concentration, $\mathrm{L}^{-3}$.

$H$ concentration distribution for vacancies, $\mathrm{L}^{-4}$.

$J \quad$ distribution of an $r_{\mathrm{s}}$-particle population flux per unit of cross-section area, $\mathrm{L}^{-3} \mathrm{~T}^{-1}$.

$\underline{J} \quad$ distribution of an $r_{\mathrm{s}}$-particle population flux through the $r_{\mathrm{p}}$-pores per unit of cross-section area, $\mathrm{L}^{-4} \mathrm{~T}^{-1}$.

$k_{0} \quad$ initial permeability, $\mathrm{L}^{2}$.

$k(\sigma)$ formation damage function, dimensionless.

$L \quad$ core length, L.

$p$ pressure, $\mathrm{M} / \mathrm{T}^{2} \mathrm{~L}$.

$P \quad$ probability of a particle with radius $r_{\mathrm{s}}$ to meet a pore with radius $r_{\mathrm{p}}$.

$r_{\mathrm{p}} \quad$ pore radius, $\mathrm{L}$.

$r_{\mathrm{s}} \quad$ particle radius, $\mathrm{L}$.

$t \quad$ dimensional time, $\mathrm{T}$.

$15 T$ dimensionless time.

${ }^{*}$ Author for Correspondences: Tel: +21-24-773+6565; Fax: +21-24-773-6564; E-mail: pavel@lenep.uenf.br 


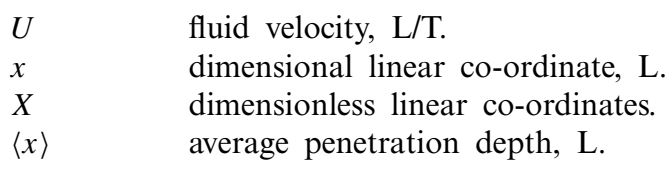

\section{1. Introduction}

18 Deep bed filtration of water with particles occurs in several industrial and environmental processes like water filtration and soil contamination. In petroleum industry, deep bed filtration of drilling fluid happens during well drilling; it also takes place near to injection wells during seawater injection causing injectivity reduction.

The particle capture in porous media can be caused by different physical mechanisms (Elimelech et al., 1995):

- size exclusion (large particles are captured in small pores and pass through large pores);

- electrical forces (London - Van der Waals, double electrical layer, etc.);

- gravity segregation;

- multi particle bridging.

30 In the current paper, the size exclusion mechanism is discussed.

A phenomenological model for the particle-capture and permeabilitydamage process was proposed by Iwasaki (1937) and used in filtration processes (Herzig, et al., 1970) and in well injectivity with rock permeability decline (Pang and Sharma, 1994; Wennberg and Sharma, 1997). The model assumes linear kinetics of particle deposition, and exhibits a good 
agreement with laboratory data. So, the model can be used for prediction purposes, like forecast of well injectivity decline based on laboratory coreflood tests. Nevertheless, the model does not distinguish between different mechanisms of formation damage. Therefore, the model cannot be used for diagnostic purposes, like determination of the dominant capture mechanism from well data.

The model predicts that the particle breakthrough happens after injection of one pore volume. Nevertheless, several cases where the breakthrough time significantly differs from one pore volume injected have been reported in the literature for particulate and polymer suspensions (Dawson and Lantz, 1972; Bartelds et al., 1997; Veerapen et al., 2001; Massei et al., 2002).

In case of size exclusion mechanism, the larger are the particles and the smaller are the pores, the more intensive is the capture and the larger is the formation damage. Nevertheless, several attempts to correlate the formation damage with sizes of particles and pores were unsuccessful (Oort et al., 1993; Bedrikovetsky et al., 2001, 2003). It could mean that either size exclusion mechanisms never dominate, or the phenomenological model for average concentrations is not general/universal enough. One of ways around this contradiction is micro scale modelling of each capture mechanism.

Different network micro models have been developed by Payatakes et al. (1973, 1974), Sahimi and Indakm (1991), Rege and Fogler (1988), (see Khilar and Fogler, 1998), Siqueira et al. (2003). Different physical mechanisms of particle retention are included in these models.

Sharma and Yortsos (1987a), derived basic population balance equations for transport of particulate suspensions in porous media. The model accounts for particle and pore size distribution variation due to different particle capture mechanisms. It is assumed that an overall pore space is accessible for particles and the particle population moves with the average flow velocity of the carrier water. In the case of porous medium with the uniform pore size distribution, this assumption results in independent deep bed filtration of different particle size populations. Nevertheless, during deep bed filtration with size exclusion mechanism, particles smaller than the pore radii should pass the rock without being captured and particles larger than the pore radii should not enter the rock.

The pore size exclusion supposes that the particles can enter just larger pores, i.e. only the fraction of porosity is accessible for particles. Therefore, the particles are carried by water flowing just via the accessible pore space, i.e. the water flux carrying particles of a fixed size is just a fraction of the overall water flux via porous media. The effects of porous space accessibility and flux reduction due to finite size of polymer molecules have been observed and mathematically described for flow of polymer solutions in rocks (Dawson and Lantz, 1972; Bartelds et al., 1997). 
In the current work, the effects of particle flux reduction and porous space inaccessibility due to selective flow of different size particles are included into the model for deep bed filtration. The terms of advective flux reduction and accessibility appear in the population balance equation. An analytical solution for the uniform pore size medium shows that deep bed filtration does not occur - large particles do not enter the porous media, and small particles move without capture.

For a small pore size variation medium, an analytical solution found shows that only intermediate size particles perform deep bed filtration. In this case, the population velocity is particle size-dependent. The averaged equations for deep bed filtration of intermediate size particles significantly differ from the classical deep bed filtration model.

In Section 2, the classical deep bed filtration equations are presented. Its stochastic generalization accounting for pore and particle size distributions and for flux reduction with pore accessibility is derived in Section 3. The initial-boundary value problem for suspension injection has a Goursat type; it allows obtaining the exact formulae for captured-particle and pore populations at the inlet cross-section without solving the initial-boundary value problem (Section 4). Section 5 contains analytical solution for a single pore size medium. Exact analytical solution and averaged equations for deep bed filtration in a media with small pore size variation are also derived in Section 6 .

\section{Classical Deep Bed Filtration Model}

The deep bed filtration system consists of equations for the particle mass balance, for the particle capture kinetics and of Darcy's law (Iwasaki, 1937; Herzig et al., 1970)

$$
\begin{aligned}
& \frac{\partial c(X, T)}{\partial T}+\frac{\partial c(X, T)}{\partial X}=-\frac{1}{\phi} \frac{\partial \sigma(X, T)}{\partial T}, \\
& \frac{\partial \sigma(X, T)}{\partial T}=\lambda(\sigma) \phi c(X, T), \\
& U=-\frac{k_{0} k(\sigma)}{\mu L} \frac{\partial p}{\partial X}
\end{aligned}
$$

where $\lambda(\sigma)=\lambda^{\prime}(\sigma) L$ is the dimensionless filtration coefficient that is equal to probability that a particle will be captured during flow through a specimen; $X$ and $T$ are dimensionless coordinate and time; $c(X, T)$ is the suspended particle concentration that is equal to the number of suspended particles per unit of pore space volume; $\sigma(X, T)$ is the deposited particle concentration that is equal to the number of retained particles per unit of 
114 porous rock volume. The formation damage function $k(\sigma)$ shows how per115 meability declines due to particle deposition.

The velocity $U$ is independent of $X$ due to suspension incompressibility. Therefore, the third equation (1) separates from the first and second equations that can be solved independently. The first and second equation (1) form the kinematics model for transport and capture of particles, the third equation is a dynamical model that predicts pressure gradient increase due to permeability decline with the particle retention.

In the case of constant filtration coefficient, the particle penetration depth equals $1 / \lambda$.

In the case of size exclusion capture, the larger are the particles and the smaller are the pores, the higher is the capture rate. Nevertheless, the phenomenological model (1) does not account for particle and pore size distributions.

In the current work, the emphasis is on the size exclusion mechanism of particle capture in the model accounting for particle and pore size distributions.

It is worth mentioning that particles move with the carrier water velocity, according to the continuity equation (1). Analytical solution for onedimensional deep bed filtration contains the suspended concentration shock that moves with the carrier water velocity, the particles appear at the core outlet after one pore volume injected and the suspended and captured concentrations are equal to zero ahead of this shock (Herzig et al., 1970).

\section{3. Governing Equations}

138 In this section we derive the population balance equations for flow of water 139 with suspended particles in porous media. In the derivations of the kinetic 140 equations, we will proceed from an assumption similar to the Boltzmann's 141 assumption about "molecular chaos" (Landau and Lifshitz, 1986). Some 142 particles are captured by the rock from the suspension by size exclusion 143 mechanism, i.e. if a large particle arrives to a small pore, $r_{\mathrm{p}}<r_{\mathrm{s}}$, it is cap144 tured and plugs the pore; otherwise, a small particle $r_{\mathrm{p}}>r_{\mathrm{s}}$ passes the pore 145 without being captured (Figure 1). It is also assumed that each particle can 146 plug only one pore, and vice versa.

147 The geometric model structure of the pore space is as follows:

148 - locally the porous space is a bundle of parallel capillary;

- the flux through each pore is proportional to the fourth power of its radius;

- the complete mixing takes place at the length scale $l$, i.e. there is a nonzero probability for a particle moving through any pore at the point $x$ to get into any pore at the point $x+l$. 


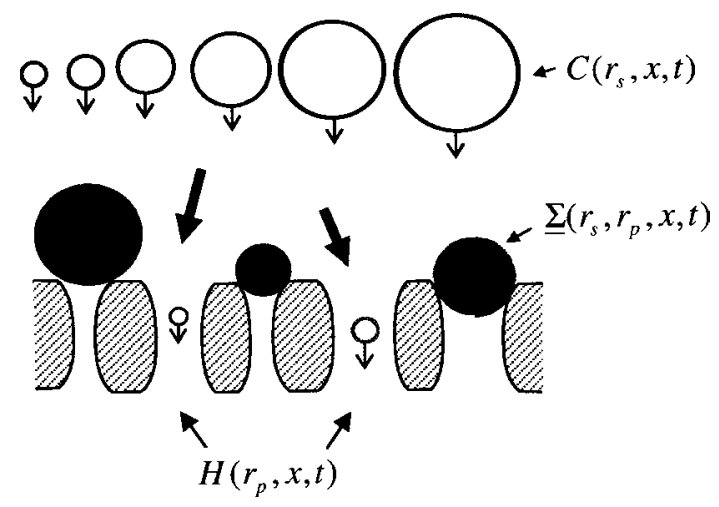

Figure 1. Schema of the large particle entrapment by small pores.

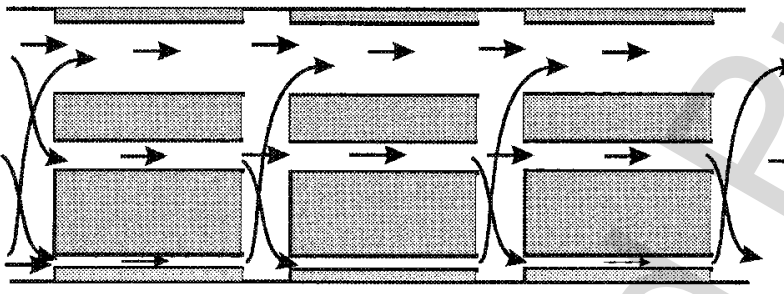

(a)

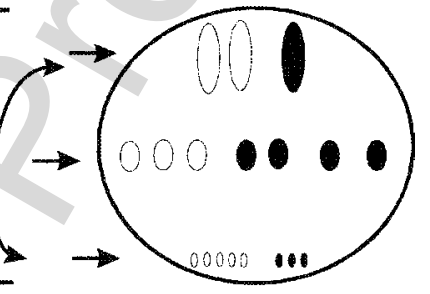

(b)

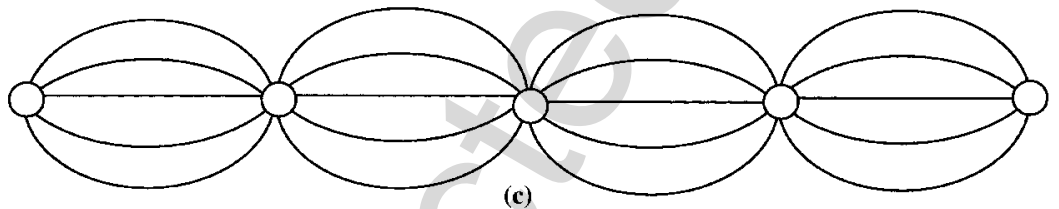

Figure 2. Separation of particle flow and capture by inserting the mixing chambers (sieves) into a capillary bundle porous media: (a) particle trajectories in capillaries and chambers, (b) frontal cross section, (c) schema for links between pores in sequential capillary bundle sections.

The example of the porous medium under consideration is shown in Figure 2(a)-(c) - it is a bundle of parallel capillary alternated by mixing chambers. The complete mixing of different size particles occurs in the chambers. The particle transport and capture occurring simultaneously in natural rocks, are separated in the proposed model. The particles move in the sections of a bundle of parallel capillary without being captured (Figure 2a). The capture occurs at the thin pore inlet, where large particles arrive. So, an inlet cross-section of each parallel capillary section acts as a sieve, i.e. large particles do not enter thin pores and are captured at chamber outlets.

It is assumed that the chamber volume is negligible if compared with the capillary (pore) volume. 
In order to describe the pore size exclusion mechanism, one should introduce distributions of suspended particles, captured particles and pores over radius

$$
\int_{0}^{\infty} f_{\mathrm{s}}\left(r_{\mathrm{s}}, x, t\right) \mathrm{d} r_{\mathrm{s}}=1, \quad \int_{0}^{\infty} f_{T}\left(r_{\mathrm{s}}, x, t\right) \mathrm{d} r_{\mathrm{s}}=1, \quad \int_{0}^{\infty} f_{\mathrm{p}}\left(r_{\mathrm{p}}, x, t\right) \mathrm{d} r_{\mathrm{p}}=1 .
$$

The product $f_{\mathrm{s}}\left(r_{\mathrm{s}}, x, t\right) \mathrm{d} r_{\mathrm{s}}$ is the fraction of particles with radii between $r_{\mathrm{s}}$ and $r_{\mathrm{s}}+\mathrm{d} r_{\mathrm{s}}$. The concentration $C\left(r_{\mathrm{s}}, x, t\right) \mathrm{d} r_{\mathrm{s}}$ of suspended particles with radii between $r_{\mathrm{s}}$ and $r_{\mathrm{s}}+\mathrm{d} r_{\mathrm{s}}$ is defined as the number of particles with radii between $r_{\mathrm{s}}$ and $r_{\mathrm{s}}+\mathrm{d} r_{\mathrm{s}}$ per unit of pore volume

$$
C\left(r_{\mathrm{s}}, x, t\right) \mathrm{d} r_{\mathrm{s}}=c(x, t) f_{\mathrm{s}}\left(r_{\mathrm{s}}, x, t\right) \mathrm{d} r_{\mathrm{s}} .
$$

Strictly speaking, $C\left(r_{\mathrm{s}}, x, t\right) \mathrm{d} r_{\mathrm{s}}$ is a concentration, and $C\left(r_{\mathrm{s}}, x, t\right)$ is a "concentration density", or "concentration distribution".

The concentration $c(x, t)$ is the total number of particles per unit of pore volume.

From Equations (2) and (3) follows that the total particle concentration is

$$
\int_{0}^{\infty} C\left(r_{\mathrm{s}}, x, t\right) \mathrm{d} r_{\mathrm{s}}=c(x, t)
$$

Let us introduce the fraction of particles with radii between $r_{\mathrm{s}}$ and $r_{\mathrm{s}}+\mathrm{d} r_{\mathrm{s}}$ have been captured by pores with radii between $r_{\mathrm{p}}$ and $r_{\mathrm{p}}+\mathrm{d} r_{\mathrm{p}}$ : $f_{T}\left(r_{\mathrm{s}}, r_{\mathrm{p}}, x, t\right) \mathrm{d} r_{\mathrm{s}} \mathrm{d} r_{\mathrm{p}}$. The particle concentration with radius $r_{\mathrm{s}}$ that have been captured by pores with radius $r_{\mathrm{p}}$ is called $\underline{\Sigma}\left(r_{\mathrm{s}}, r_{\mathrm{p}}, x, t\right)$ (Figure 1):

$$
\underline{\Sigma}\left(r_{\mathrm{s}}, r_{\mathrm{p}}, x, t\right) \mathrm{d} r_{\mathrm{p}} \mathrm{d} r_{\mathrm{s}}=\sigma(x, t) \underline{f_{T}}\left(r_{\mathrm{s}}, r_{\mathrm{p}}, x, t\right) \mathrm{d} r_{\mathrm{p}} \mathrm{d} r_{\mathrm{s}} .
$$

The product $\underline{\Sigma}\left(r_{\mathrm{s}}, r_{\mathrm{p}}, x, t\right) \mathrm{d} r_{\mathrm{s}} \mathrm{d} r_{\mathrm{p}}$ is equal to the number of particles with radii between $r_{\mathrm{s}}$ and $r_{\mathrm{s}}+\mathrm{d} r_{\mathrm{s}}$ which have been captured by pores with radii between $r_{\mathrm{p}}$, and $r_{\mathrm{p}}+\mathrm{d} r_{\mathrm{p}}$ per unit of the rock volume.

The total retained concentration $\sigma(x, t)$ is equal to the number of particles captured in a unitary volume of a porous medium.

The size exclusion capture mechanism assumes that the " $r_{\mathrm{s}}$ " particle is captured by the " $r_{\mathrm{p}}$ " pore if $r_{\mathrm{s}}>r_{\mathrm{p}}$. Therefore, $\underline{\Sigma}\left(r_{\mathrm{s}}, r_{\mathrm{p}}, x, t\right)=0$ for $r_{\mathrm{s}}<r_{\mathrm{p}}$, and the fraction of captured particles with radii between $r_{\mathrm{s}}$ and $r_{\mathrm{s}}+\mathrm{d} r_{\mathrm{s}}$ is

$$
f_{T}\left(r_{\mathrm{s}}, x, t\right) \mathrm{d} r_{\mathrm{s}}=\left[\int_{0}^{r_{\mathrm{s}}} \underline{f_{T}}\left(r_{\mathrm{s}}, r_{\mathrm{p}}, x, t\right) \mathrm{d} r_{\mathrm{p}}\right] \mathrm{d} r_{\mathrm{s}}
$$


196

Integrating (5) in $r_{\mathrm{p}}$ and accounting for (6), we obtain the concentration of captured particles with radius in the interval $\left[r_{\mathrm{s}}, r_{\mathrm{s}}+\mathrm{d} r_{\mathrm{s}}\right]$ :

$$
\left[\int_{0}^{r_{\mathrm{s}}} \underline{\Sigma}\left(r_{\mathrm{s}}, r_{\mathrm{p}}, x, t\right) \mathrm{d} r_{\mathrm{p}}\right] \mathrm{d} r_{\mathrm{s}}=\Sigma\left(r_{\mathrm{s}}, x, t\right) \mathrm{d} r_{\mathrm{s}}
$$

$$
\text { From (5)-(7) follows that }
$$

$$
\Sigma\left(r_{\mathrm{s}}, x, t\right) \mathrm{d} r_{\mathrm{s}}=\sigma(x, t) f_{T}\left(r_{\mathrm{s}}, x, t\right) \mathrm{d} r_{\mathrm{s}} .
$$

Integration of (7) in $r_{\mathrm{s}}$ from zero to infinity results in the total captured particle concentration:

$$
\int_{0}^{\infty} \Sigma\left(r_{\mathrm{s}}, x, t\right) \mathrm{d} r_{\mathrm{s}}=\sigma(x, t) .
$$

The vacant pore concentration $H\left(r_{\mathrm{p}}, x, t\right) \mathrm{d} r_{\mathrm{p}}$ with radius in the interval $\left[r_{\mathrm{p}}, r_{\mathrm{p}}+\mathrm{d} r_{\mathrm{p}}\right]$ is defined as

$$
H\left(r_{\mathrm{p}}, x, t\right) \mathrm{d} r_{\mathrm{p}}=h(x, t) f_{\mathrm{p}}\left(r_{\mathrm{p}}, x, t\right) \mathrm{d} r_{\mathrm{p}},
$$

where the total vacant pore concentration is

$$
\int_{0}^{\infty} H\left(r_{\mathrm{p}}, x, t\right) \mathrm{d} r_{\mathrm{p}}=h(x, t) .
$$

It is assumed that a captured particle plugs one pore only, and vice versa. Besides, the size exclusion mechanism assumes that an $r_{\mathrm{s}}$-particle can be captured by an $r_{\mathrm{p}}$-pore if $r_{\mathrm{s}}>r_{\mathrm{p}}$, so $\Sigma\left(r_{\mathrm{s}}, r_{\mathrm{p}}, x, t\right)=0$ for $r_{\mathrm{s}}<r_{\mathrm{p}}$. Therefore, the variation on the total number of pores with radii in the interval $\left[r_{\mathrm{p}}, r_{\mathrm{p}}+\mathrm{d} r_{\mathrm{p}}\right]$ is equal to the total number of particles captured in pores with size in the interval $\left[r_{\mathrm{p}}, r_{\mathrm{p}}+\mathrm{d} r_{\mathrm{p}}\right]$ :

$$
H\left(r_{\mathrm{p}}, x, t\right) \mathrm{d} r_{\mathrm{p}}=H\left(r_{\mathrm{p}}, x, 0\right) \mathrm{d} r_{\mathrm{p}}-\left[\int_{r_{\mathrm{s}}}^{\infty} \underline{\Sigma}\left(r_{\mathrm{s}}, r_{\mathrm{p}}, x, t\right) \mathrm{d} r_{\mathrm{s}}\right] \mathrm{d} r_{\mathrm{p}} .
$$

Differentiation of (12) with respect to $t$ results in

$$
\frac{\partial H\left(r_{\mathrm{p}}, x, t\right)}{\partial t}=-\int_{r_{\mathrm{p}}}^{\infty} \frac{\partial \underline{\Sigma}\left(r_{\mathrm{s}}, r_{\mathrm{p}}, x, t\right)}{\partial t} \mathrm{~d} r_{\mathrm{s}} .
$$

Equation (13) means that plugging of a pore is caused by the capture of whatever larger particle. 
Let us derive the population balance for suspended and captured particles.

A particle with radius $r_{\mathrm{s}}$ passes through the pore with radius $r_{\mathrm{p}}$ only if the particle radius is smaller than the pore radius, $r_{\mathrm{s}}<r_{\mathrm{p}}$. Therefore, small pores $\left(r_{\mathrm{p}}<r_{\mathrm{s}}\right)$ are inaccessible for large particles. Particles flow in larger pores only, i.e. in an accessible pore volume. Assuming that locally the pore space is a bundle of parallel capillary, we introduce the accessibility factor $\gamma$ for particles with radius $r_{\mathrm{s}}$ as a fraction of pore volume with capillary radii larger than $r_{\mathrm{s}}$ :

$$
\gamma\left(r_{\mathrm{s}}, x, t\right)=\frac{\int_{r_{\mathrm{p}}}^{\infty} r_{\mathrm{p}}^{2} H\left(r_{\mathrm{p}}, x, t\right) \mathrm{d} r_{\mathrm{p}}}{\int_{0}^{\infty} r_{\mathrm{p}}^{2} H\left(r_{\mathrm{p}}, x, t\right) \mathrm{d} r_{\mathrm{p}}} .
$$

Consequently, particles with radius $r_{\mathrm{s}}$ move in the $\gamma\left(r_{\mathrm{s}}, x, t\right)$-th fraction of pore volume.

Let us define the flux $J\left(r_{\mathrm{s}}, r_{\mathrm{p}}, x, t\right) \mathrm{d} r_{\mathrm{s}} / \mathrm{d} r_{\mathrm{p}}$ of particles with specific radius $r_{\mathrm{s}}$ via pores with a specific radius $r_{\mathrm{p}}$ and also the total flux $J\left(r_{\mathrm{s}}, x, t\right) \mathrm{d} r_{\mathrm{s}}$ of particles with radii in the interval $\left[r_{\mathrm{s}}, r_{\mathrm{s}}+\mathrm{d} r_{\mathrm{s}}\right]$. From the assumption that locally the pore space is a bundle of parallel capillary, we obtain:

$$
J\left(r_{\mathrm{s}}, x, t\right) \mathrm{d} r_{\mathrm{s}}=\left[\int_{r_{\mathrm{s}}}^{\infty} \underline{J}\left(r_{\mathrm{s}}, r_{\mathrm{p}}, x, t\right) \mathrm{d} r_{\mathrm{p}}\right] \mathrm{d} r_{\mathrm{s}} .
$$

The flux of particles with radius $r_{\mathrm{s}}$ via pores with smaller radius $\left(r_{\mathrm{p}}<r_{\mathrm{s}}\right)$ equals zero. Nevertheless, water flows via pores of all sizes including thin pores. Therefore, the water flux carrying $r_{\mathrm{s}}$-particles is lower than the overall water flux in the porous medium.

We assume that the flux via the pore $r_{\mathrm{p}}$ is proportional to the fourth power of the capillary radius $r_{\mathrm{p}}^{4}$ (Hagen-Poiseuille formula, see Landau and Lifshitz, 1987). Consequently, the fraction of the flux via pores with radii varying from $r_{\mathrm{p}}$ to $r_{\mathrm{p}}+\mathrm{d} r_{\mathrm{p}}$ is

$$
F\left(r_{\mathrm{p}}, x, t\right) \mathrm{d} r_{\mathrm{p}}=\frac{H\left(r_{\mathrm{p}}, x, t\right) r_{\mathrm{p}}^{4} \mathrm{~d} r_{\mathrm{p}}}{\int_{0}^{\infty} H\left(r_{\mathrm{p}}, x, t\right) r_{\mathrm{p}}^{4} \mathrm{~d} r_{\mathrm{p}}} .
$$

The flux of particles with specific radius $r_{\mathrm{s}}$ via pores with specific radius $r_{\mathrm{p}}$ equals the total flux of particles with radius $r_{\mathrm{s}}$ times fraction of the total flux via the pores with radius $r_{\mathrm{p}}$ only:

$$
\underline{J}\left(r_{\mathrm{s}}, r_{\mathrm{p}}, x, t\right) \mathrm{d} r_{\mathrm{s}} \mathrm{d} r_{\mathrm{p}}=U C\left(r_{\mathrm{s}}, x, t\right) \frac{H\left(r_{\mathrm{p}}, x, t\right) r_{\mathrm{p}}^{4} \mathrm{~d} r_{\mathrm{p}}}{\int_{0}^{\infty} H\left(r_{\mathrm{p}}, x, t\right) r_{\mathrm{p}}^{4} \mathrm{~d} r_{\mathrm{p}}} \mathrm{d} r_{\mathrm{s}} .
$$


The above explanation of (17) would become more rigorous by substituting the terms "specific radius" $r_{\mathrm{s}}$ and $r_{\mathrm{p}}$ by the terms "in the intervals" $\left[r_{\mathrm{s}}, r_{\mathrm{s}}+\mathrm{d} r_{\mathrm{s}}\right]$ and $\left[r_{\mathrm{p}}, r_{\mathrm{p}}+\mathrm{d} r_{\mathrm{p}}\right]$, respectively.

The total flux $J\left(r_{\mathrm{s}}, x, t\right) \mathrm{d} r_{\mathrm{s}}$ of particles with radii in the interval $\left[r_{\mathrm{s}}, r_{\mathrm{s}}+\right.$ $\mathrm{d} r_{\mathrm{s}}$ ] accounts for transport via all pores with radius larger than $r_{\mathrm{s}}$ :

$$
J\left(r_{\mathrm{s}}, x, t\right) \mathrm{d} r_{\mathrm{s}}=U C\left(r_{\mathrm{s}}, x, t\right) \frac{\int_{r_{\mathrm{s}}}^{\infty} H\left(r_{\mathrm{p}}, x, t\right) r_{\mathrm{p}}^{4} \mathrm{~d} r_{\mathrm{p}}}{\int_{0}^{\infty} H\left(r_{\mathrm{p}}, x, t\right) r_{\mathrm{p}}^{4} \mathrm{~d} r} \mathrm{~d} r_{\mathrm{s}} .
$$

Introducing the fraction of the total flux that carries particles with radius $r_{\mathrm{s}}$

$$
\alpha\left(r_{\mathrm{s}}, x, t\right)=\frac{\int_{r_{\mathrm{s}}}^{\infty} r_{\mathrm{p}}^{4} H\left(r_{\mathrm{p}}, x, t\right) \mathrm{d} r_{\mathrm{p}}}{\int_{0}^{\infty} r_{\mathrm{p}}^{4} H\left(r_{\mathrm{p}}, x, t\right) \mathrm{d} r_{\mathrm{p}}}
$$

from (18) and (19), we obtain the following formula for the flux of particles with radii varying from $r_{\mathrm{s}}$ to $r_{\mathrm{s}}+\mathrm{d} r_{\mathrm{s}}$ :

$$
J\left(r_{\mathrm{s}}, x, t\right) \mathrm{d} r_{\mathrm{s}}=U \alpha\left(r_{\mathrm{s}}, x, t\right) C\left(r_{\mathrm{s}}, x, t\right) \mathrm{d} r_{\mathrm{s}}
$$

From now on, $\alpha$ will be called the flux reduction factor.

Formulae for the flux reduction and accessibility factors ((14) and (19)) can be derived for regular pore networks using effective medium or percolation theories (Sharma and Yortsos, 1987b,c; Seljakov and Kadet, 1996). From either theory will follow two threshold values for the flux reduction factor corresponding to existence of infinite clusters for small and for large particles.

In the case of low concentrated suspensions, the pore space fraction occupied by retained particles is negligibly small if compared with the overall pore space. Therefore, the porosity is assumed to be constant.

From now on, we consider concentration densities instead of concentrations, so the multipliers $\mathrm{d} r_{\mathrm{s}}$ and $\mathrm{d} r_{\mathrm{p}}$ in both sides of equations are dropped. In this case, the equation for particle number balance for $r_{\mathrm{s}}$-population accounting for retention is

$$
\phi \frac{\partial\left[\gamma\left(r_{\mathrm{s}}, x, t\right) C\left(r_{\mathrm{s}}, x, t\right)\right]}{\partial t}+\frac{\partial J\left(r_{\mathrm{s}}, x, t\right)}{\partial x}=-\frac{\partial \Sigma\left(r_{\mathrm{s}}, x, t\right)}{\partial t} .
$$

Substitution of (20) into (21) results in the following form of the population balance equation:

$$
\phi \frac{\partial\left[\gamma\left(r_{\mathrm{s}}, x, t\right) C\left(r_{\mathrm{s}}, x, t\right)\right]}{\partial t}+\frac{\partial\left[U \alpha\left(r_{\mathrm{s}}, x, t\right) C\left(r_{\mathrm{s}}, x, t\right)\right]}{\partial x}=-\frac{\partial \Sigma\left(r_{\mathrm{s}}, x, t\right)}{\partial t} .
$$

In order to obtain a closed system of governing equations, let us derive equations for particle capture and pore plugging rates. The probability $P$ 
282

283

284

285

286

of a particle with radius from the interval $\left[r_{\mathrm{s}}, r_{\mathrm{s}}+\mathrm{d} r_{\mathrm{s}}\right]$ to meet a pore with radius from the interval $\left[r_{\mathrm{p}}, r_{\mathrm{p}}+\mathrm{d} r_{\mathrm{p}}\right]$ is proportional to the product between the number of particles with radius from the interval $\left[r_{\mathrm{s}}, r_{\mathrm{s}}+\mathrm{d} r_{\mathrm{s}}\right]$ and the flux fraction that passes via the pores with radius from the interval $\left[r_{\mathrm{p}}, r_{\mathrm{p}}+\right.$ $\mathrm{d} r_{\mathrm{p}}$ ] (Herzig et al., 1970):

$$
P \propto U C\left(r_{\mathrm{s}}, x, t\right) \mathrm{d} r_{\mathrm{s}} \frac{r_{\mathrm{p}}^{4} H\left(r_{\mathrm{p}}, x, t\right) \mathrm{d} r_{\mathrm{p}}}{\int_{0}^{\infty} r_{\mathrm{p}}^{4} H\left(r_{\mathrm{p}}, x, t\right) \mathrm{d} r_{\mathrm{p}}} .
$$

The number of particles with size in the interval $\left[r_{\mathrm{s}}, r_{\mathrm{s}}+\mathrm{d} r_{\mathrm{s}}\right]$ captured in pores with radius in the interval $\left[r_{\mathrm{p}}, r_{\mathrm{p}}+\mathrm{d} r_{\mathrm{p}}\right]$ per unit of time is called the particle-capture rate. This rate is proportional to the probability $P$, (23), and the proportionality co-efficient is called the filtration co-efficient $-\lambda^{\prime}\left(r_{\mathrm{s}}, r_{\mathrm{p}}\right)$ :

$$
\frac{\partial \underline{\Sigma}\left(r_{\mathrm{s}}, r_{\mathrm{p}}, x, t\right)}{\partial t}=\lambda^{\prime}\left(r_{\mathrm{s}}, r_{\mathrm{p}}\right) U C\left(r_{\mathrm{s}}, x, t\right) \frac{r_{\mathrm{p}}^{4} H\left(r_{\mathrm{p}}, x, t\right)}{\int_{0}^{\infty} r_{\mathrm{p}}^{4} H\left(r_{\mathrm{p}}, x, t\right) \mathrm{d} r_{\mathrm{p}}} .
$$

Here, as in the majority of following formulae, we omitted $\mathrm{d} r_{\mathrm{s}} / \mathrm{d} r_{\mathrm{p}}$ in both sides of (24). It means that we will work with concentrations density $(C, \Sigma$ and $H)$ instead of concentrations $\left(C \mathrm{~d} r_{\mathrm{s}}, \Sigma \mathrm{d} r_{\mathrm{s}}\right.$, and $\left.H-\mathrm{d} r_{\mathrm{p}}\right)$.

The filtration coefficient is equal to zero for the absence of capture:

$$
\lambda^{\prime}\left(r_{\mathrm{s}}, r_{\mathrm{p}}\right)=0: \quad r_{\mathrm{p}}>r_{\mathrm{s}}
$$

Integration of both sides of (24) over $r_{\mathrm{p}}$ from zero to infinity and accounting for (25), results in the expression for the total capture rate of particles with radius $r_{\mathrm{s}}$ :

$$
\frac{\partial \Sigma\left(r_{\mathrm{s}}, x, t\right)}{\partial t}=\frac{U C\left(r_{\mathrm{s}}, x, t\right)}{\int_{0}^{\infty} r_{\mathrm{p}}^{4} H\left(r_{\mathrm{p}}, x, t\right) \mathrm{d} r_{\mathrm{p}}} \int_{0}^{r_{\mathrm{s}}} \lambda^{\prime}\left(r_{\mathrm{s}}, r_{\mathrm{p}}\right) H\left(r_{\mathrm{p}}, x, t\right) \mathrm{d} r_{\mathrm{p}}
$$

Substituting the capture rate (24) into (13), we obtain the equation for pore plugging kinetics

$$
\frac{\partial H\left(r_{\mathrm{p}}, x, t\right)}{\partial t}=\frac{U H\left(r_{\mathrm{p}}, x, t\right) r_{\mathrm{p}}^{4}}{\int_{0}^{\infty} H\left(r_{\mathrm{p}}, x, t\right) r_{\mathrm{p}}^{4} \mathrm{~d} r_{\mathrm{p}}} \int_{r_{\mathrm{p}}}^{\infty} \lambda^{\prime}\left(r_{\mathrm{s}}, r_{\mathrm{p}}\right) C\left(r_{\mathrm{s}}, x, t\right) \mathrm{d} r_{\mathrm{s}}
$$

It is assumed that the aqueous suspension is incompressible, the total flux conserves, $U=U(t)$, and term $U$ can be taken out of $x$-derivative in Equation (22). 
Equations (22), (26) and (27) form a closed system for three unknowns $C\left(r_{\mathrm{s}}, x, t\right), \Sigma\left(r_{\mathrm{s}}, x, t\right)$ and $H\left(r_{\mathrm{p}}, x, t\right)$ :

$$
\begin{aligned}
& \phi \frac{\partial\left[\gamma\left(r_{\mathrm{s}}, x, t\right) C\left(r_{\mathrm{s}}, x, t\right)\right]}{\partial t}+U \frac{\partial\left[\alpha\left(r_{\mathrm{s}}, x, t\right) C\left(r_{\mathrm{s}}, x, t\right)\right]}{\partial x}=-\frac{\partial \Sigma\left(r_{\mathrm{s}}, x, t\right)}{\partial t}, \\
& \frac{\partial \Sigma\left(r_{\mathrm{s}}, x, t\right)}{\partial t}=U C\left(r_{\mathrm{s}}, x, t\right) \frac{\int_{0}^{r_{\mathrm{s}}} \lambda^{\prime}\left(r_{\mathrm{s}}, r_{\mathrm{p}}\right) r_{\mathrm{p}}^{4} H\left(r_{\mathrm{p}}, x, t\right) \mathrm{d} r_{\mathrm{p}}}{\int_{0}^{\infty} r_{\mathrm{p}}^{4} H\left(r_{\mathrm{p}}, x, t\right) \mathrm{d} r_{\mathrm{p}}}, \\
& \frac{\partial H\left(r_{\mathrm{p}}, x, t\right)}{\partial t}=-U \frac{r_{\mathrm{p}}^{4} H\left(r_{\mathrm{p}}, x, t\right)}{\int_{0}^{\infty} r_{\mathrm{p}}^{4} H\left(r_{\mathrm{p}}, x, t\right) \mathrm{d} r_{\mathrm{p}}} \int_{r_{\mathrm{p}}}^{\infty} \lambda^{\prime}\left(r_{\mathrm{s}}, r_{\mathrm{p}}\right) C\left(r_{\mathrm{s}}, x, t\right) \mathrm{d} r_{\mathrm{s}} .
\end{aligned}
$$

Introduction of dimensionless variables

$$
x=\frac{x}{L}, \quad T=\frac{U T}{L \phi}, \quad \lambda=\lambda^{\prime} L,
$$

transforms the system (28) to the form:

$$
\begin{aligned}
& \frac{\partial\left[\gamma\left(r_{\mathrm{s}}, X, T\right) C\left(r_{\mathrm{s}}, X, T\right)\right]}{\partial T}+U \frac{\partial\left[\alpha\left(r_{\mathrm{s}}, X, T\right) C\left(r_{\mathrm{s}}, X, T\right)\right]}{\partial X} \\
& =-\frac{1}{\phi} \frac{\partial \Sigma\left(r_{\mathrm{s}}, X, T\right)}{\partial T}, \\
& \frac{\partial \Sigma\left(r_{\mathrm{s}}, X, T\right)}{\partial T}=\phi C\left(r_{\mathrm{s}}, X, T\right) \frac{\int_{0}^{r_{\mathrm{s}}} \lambda\left(r_{\mathrm{s}}, r_{\mathrm{p}}\right) r_{\mathrm{p}}^{4} H\left(r_{\mathrm{p}}, X, T\right) \mathrm{d} r_{\mathrm{p}}}{\int_{0}^{\infty} r_{\mathrm{p}}^{4} H\left(r_{\mathrm{p}}, X, T\right) \mathrm{d} r_{\mathrm{p}}}, \\
& \frac{\partial H\left(r_{\mathrm{p}}, X, T\right)}{\partial T}=-\phi \frac{r_{\mathrm{p}}^{4} H\left(r_{\mathrm{p}}, X, T\right)}{\int_{0}^{\infty} r_{\mathrm{p}}^{4} H\left(r_{\mathrm{p}}, X, T\right) \mathrm{d} r_{\mathrm{p}}} \int_{r_{\mathrm{p}}}^{\infty} \lambda\left(r_{\mathrm{s}}, r_{\mathrm{p}}\right) C\left(r_{\mathrm{s}}, X, T\right) \mathrm{d} r_{\mathrm{s}} .
\end{aligned}
$$

Boundary condition at the core inlet corresponds to injection of water with a given particle size distribution $C^{(0)}\left(r_{\mathrm{s}}, T\right)$. The injected $r_{\mathrm{s}}$-particle flux is equal to $C^{(0)}\left(r_{\mathrm{s}}, T\right) U$. The inlet core/reservoir cross-section acts as a sieve. The injected $r_{\mathrm{s}}$-particles are carried into the porous medium by a fraction of the water flux via accessible pores $-\alpha^{(0)}\left(r_{\mathrm{s}}, T\right) U$ (Figure 2(b)). The injected $r_{\mathrm{s}}$-particles carried by water flux via inaccessible pores [1$\left.\alpha^{0}\left(r_{\mathrm{s}}, T\right)\right] U$ are deposited at the outer surface of the inlet and form the external filter cake from the very beginning of injection. For particles larger than any pore, there is no accessible pores and flux reduction factor is zero, $\alpha^{(0)}\left(r_{\mathrm{s}}, T\right)=0$. So, all these particles are retained at the inlet cross-section, contributing to external filter cake growth. On the other hand, for particles smaller than the smallest pore, $\alpha^{(0)}\left(r_{\mathrm{s}}, T\right)=1$. So, all these particles enter porous medium without being captured.

The density of the $r_{\mathrm{s}}$-particle flux entering porous medium (in situ $r_{\mathrm{s}}$-particle flux) is equal to $C^{(0)}\left(r_{\mathrm{s}}, T\right) \alpha^{(0)}\left(r_{\mathrm{s}}, T\right) U$; and the fraction captured 
at the inlet cross-section is equal to $C^{(0)}\left(r_{\mathrm{s}}, T\right)\left[1-\alpha^{(0)}\left(r_{\mathrm{s}}, T\right)\right] U$. Therefore, the $r_{\mathrm{S}}$-particle concentration is continuous at $X=0$.

We also assume that the retained at the outer surface of the inlet large particles do not restrict access of newly arriving particles to the core inlet before the transition time (Khatib, 1994; Pang and Sharma, 1994). The external cake does not form a solid matrix before the transition time and cannot capture the particles from the injected suspension.

Initial condition corresponds to the absence of either suspended or captured particles in porous media before the flow. Finally,

$$
\begin{aligned}
& X=0: C\left(r_{\mathrm{s}}, 0, T\right)=C^{(0)}\left(r_{\mathrm{s}}, T\right), \\
& T=0: C\left(r_{\mathrm{s}}, X, 0\right)=0, \quad \Sigma\left(r_{\mathrm{s}}, X, 0\right)=0, \quad H\left(r_{\mathrm{p}}, X, 0\right)=H_{0}\left(r_{\mathrm{p}}, X\right) .
\end{aligned}
$$

Integration of (13) in $r_{\mathrm{p}}$, from zero to infinity results in a conservation law for pore number

$$
\frac{\partial h}{\partial T}=-\frac{\partial \sigma}{\partial T},
$$

which leads to

$$
h(X, T)=h_{0}(X)-\sigma(X, T) .
$$

Equation (33) shows that one particle can plug only one pore and vice versa.

\section{Particle and Pore Populations at the Inlet Cross-Section}

Second and third equations of system (30) do not contain $X$-derivative, so it is not necessary to set the corresponding species concentrations at the inlet boundary $X=0$ (The so-called Goursat problem; Tikhonov and Samarskii, 1990). It means that one do not fix the injected concentration of an immobile specie, i.e. retained particles and vacancies. Nevertheless, these values can be calculated using boundary conditions for mobile species and the kinetic equations for immobile species (second and third equation of system (30)).

Let us fix $X=0$ in system (30) and substitute the boundary condition (31) into second and third equations of system (30). Finally, we obtain the system of two ordinary integro-differential equations for captured particle and vacant pore concentrations at the plug inlet:

$$
\begin{aligned}
& \frac{\mathrm{d} \Sigma^{(0)}\left(r_{\mathrm{s}}, T\right)}{\mathrm{d} T}=\phi C^{(0)}\left(r_{\mathrm{s}}, T\right) \frac{\int_{0}^{r_{\mathrm{s}}} \lambda\left(r_{\mathrm{s}}, r_{\mathrm{p}}\right) r_{\mathrm{p}}^{4} H^{(0)}\left(r_{\mathrm{p}}, T\right) \mathrm{d} r_{\mathrm{p}}}{\int_{0}^{\infty} r_{\mathrm{p}}^{4} H^{(0)}\left(r_{\mathrm{p}}, T\right) \mathrm{d} r_{\mathrm{p}}}, \\
& \frac{\mathrm{d} H^{(0)}\left(r_{\mathrm{p}}, T\right)}{\mathrm{d} T}=-\phi \frac{r_{\mathrm{p}}^{4} H^{(0)}\left(r_{\mathrm{p}}, T\right)}{\int_{0}^{\infty} r_{\mathrm{p}}^{4} H^{(0)}\left(r_{\mathrm{p}}, T\right) \mathrm{d} r_{\mathrm{p}}} \int_{r_{\mathrm{p}}}^{\infty} \lambda\left(r_{\mathrm{s}}, r_{\mathrm{p}}\right) C^{(0)}\left(r_{\mathrm{s}}, T\right) \mathrm{d} r_{\mathrm{s}},
\end{aligned}
$$


where,

$$
H^{(0)}\left(r_{\mathrm{p}}, T\right)=H\left(r_{\mathrm{p}}, X=0, T\right), \quad \Sigma^{(0)}\left(r_{\mathrm{s}}, T\right)=\Sigma\left(r_{\mathrm{s}}, X=0, T\right) .
$$

The second equation (34) is independent of the first equation, and can be solved separately. Afterwards, the first equation allows calculating the deposition kinetics.

There were no deposited particles and plugged pores at the beginning of deep bed filtration. It provides the initial conditions for the system of ordinary integro-differential Equations (34).

$$
\Sigma^{(0)}\left(r_{\mathrm{p}}, T=0\right)=0, \quad H^{(0)}\left(r_{\mathrm{p}}, T=0\right)=H_{0}^{(0)}\left(r_{\mathrm{p}}\right) .
$$

The solution of the second ordinary integro-differential Equation (34) allows calculating the transition time $\left(T_{\mathrm{tr}}\right)$ from the system of deep bed filtration in porous media. The filtration at the inlet cross-section stops at the moment when the concentration of vacancies $H^{(0)}\left(r_{\mathrm{p}}, T\right)$ forming an infinite cluster decreases up to percolation threshold.

The solution $H^{(0)}\left(r_{\mathrm{p}}, T\right)$ results in calculation of the $r_{\mathrm{s}}$-particle flux $C^{(0)}\left(r_{\mathrm{s}}, T\right)\left[1-\alpha^{(0)}\left(r_{\mathrm{s}}, T\right)\right] U$ forming an external filter cake from the very beginning of the particle injection. It allows describing the external filter cake formation before the transition time, when particles still penetrate into porous medium.

\section{5. Filtration in a Single Pore Size Medium}

384 Consider the injection of suspension with any given particle size distribution in a porous medium with a single pore radius $r_{\mathrm{p}}^{\prime}$ :

$$
H\left(r_{\mathrm{p}}, X, T\right)=h(X, T) \delta\left(r_{\mathrm{p}}-r_{\mathrm{p}}^{\prime}\right) .
$$

Figure 3(a) shows the pore size distribution (Dirac's delta function) at $T=0$ and the particle size distribution in the injected suspension at $X=0$.

Let us first consider propagation of small particles with $r_{\mathrm{s}}<r_{\mathrm{p}}^{\prime}$. For this case, formulae (14) and (19) show that $\alpha=\gamma=1$; i.e. all pores are accessible for small particles, and there is no flux reduction.

Substitution of the pore size distribution (37) into (30) results in the following system for deep bed filtration of small particles:

$$
\begin{aligned}
& \frac{\partial C\left(r_{\mathrm{s}}, X, T\right)}{\partial T}+\frac{\partial C\left(r_{\mathrm{s}}, X, T\right)}{\partial X}=0, \\
& \frac{\partial \Sigma\left(r_{\mathrm{s}}, X, T\right)}{\partial T}=0 .
\end{aligned}
$$



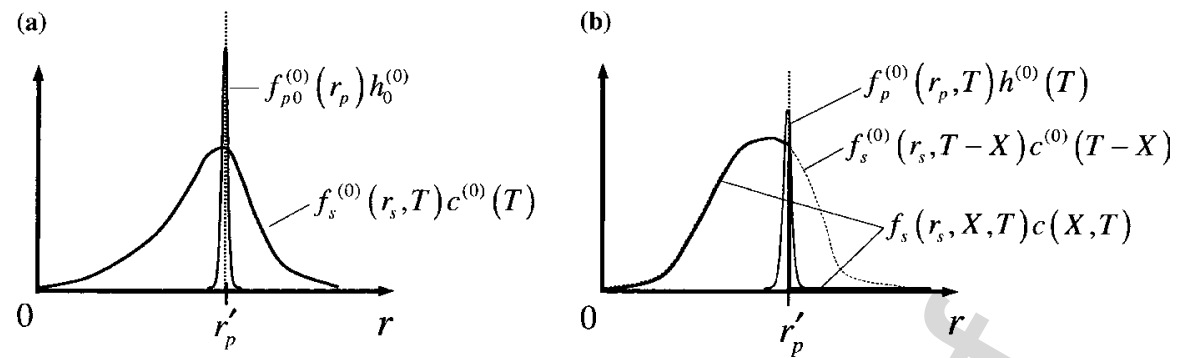

Figure 3. Distributions of suspended particles and pores in a single pore size medium: (a) initial and boundary concentration distributions for pores and suspended particles, respectively; (b) particle distribution for any $X$ and $T$ (continuous curve) and at $X=0$ (dashed curve); pore distribution at the inlet cross-section for $T>0$.

The solution of a linear hyperbolic equation (first Equation (38)) subject to initial-boundary conditions (31) is a travelling wave:

$$
C\left(r_{\mathrm{s}}, X, T\right)= \begin{cases}C^{(0)}\left(r_{\mathrm{s}}, T-X\right), & X<T \\ 0, & X>T .\end{cases}
$$

Therefore, small particles are transported with the velocity of carrier water without being trapped. There are no suspended particles ahead of the injected water front. Particle distribution profile behind the front moves with unitary velocity along the porous medium. It repeats the shape of the injected concentration $C^{(0)}\left(r_{\mathrm{s}}, T\right)$ with delay that equals $X$.

We consider the case where there were no trapped particles in porous medium before the injection (initial condition (31)). As it follows from the second Equation (38), the capture of small particles does not happen. Consequently, for any $T \geqslant 0$

$$
\Sigma\left(r_{\mathrm{s}}, X, T\right)=0 .
$$

Therefore, no pores will be plugged by small particles.

Now consider propagation of large particles $\left(r_{\mathrm{s}}>r_{\mathrm{p}}^{\prime}\right)$. In this case, from (14) and (19) follows that $\alpha=\gamma=0$.

Therefore, none of pores is accessible for large particles, and there is no large particle flux.

Substitution of (37) into (30) results in the following system: 
417 From initial condition (31) and first Equation (41) follows that

$418 \quad \Sigma\left(r_{\mathrm{s}}, X, T\right)=0$,

419 i.e. no large particles are deposited in the reservoir.

420 From first equation (34) we obtain the captured particle concentration 421 at the core inlet:

$$
\Sigma^{(0)}\left(r_{\mathrm{s}}, T\right)=\lambda\left(r_{\mathrm{s}}, r_{\mathrm{p}}^{\prime}\right) \phi \int_{0}^{T} C^{(0)}\left(r_{\mathrm{s}}, T\right) \mathrm{d} T .
$$

Therefore, all large particles are captured at the inlet cross-section.

It is assumed that there were no suspended particles before the injection (initial condition (31)). In this case, from first and second equation (41) follows that:

$$
C\left(r_{\mathrm{s}}, X, T\right)=0: X>0,
$$

i.e. no large particles $\left(r_{\mathrm{s}}>r_{\mathrm{p}}^{\prime}\right)$ enter the reservoir.

Substituting (44) into third equation (41) and solving the resulting ordi430 nary differential equation, accounting for initial and boundary conditions 431 (31), we obtain:

$$
h(X, T)=h_{0}(X): X>0,
$$

433 i.e. the number of vacant pores does not change during the injection.

The line 2 in Figure 4 shows that large particles never arrive to the core outlet. It was also observed in laboratory study (Massei et al., 2002), where size exclusion was the dominant capture mechanism.

Now let us study accumulation of large particles at the core inlet. 438 Substituting (43) into (9), accounting for (44), results in:

$$
\sigma^{(0)}(T)=\phi \int_{r_{\mathrm{p}}^{\prime}}^{\infty} \lambda\left(r_{\mathrm{s}}, r_{\mathrm{p}}^{\prime}\right) \int_{0}^{T} C^{(0)}\left(r_{\mathrm{s}}, \tau\right) \mathrm{d} \tau \mathrm{d} r_{\mathrm{s}}
$$

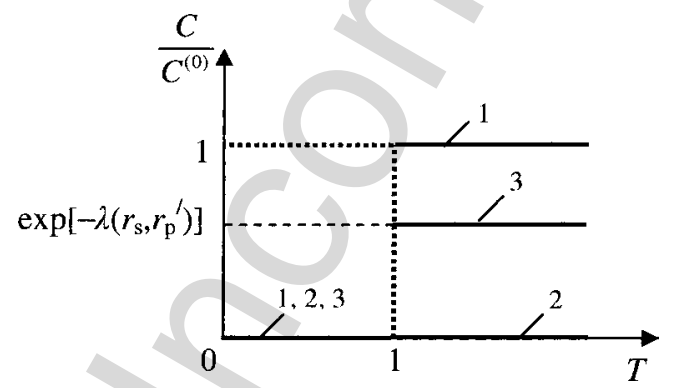

Figure 4. Breakthrough curves for different size particles (at $X=1$ ): 1 - for particles smaller than $r_{\mathrm{p}}^{\prime}$ by the proposed model 2 - for particles larger than $r_{\mathrm{p}}^{\prime}$ by the proposed model 3 - for particles larger than $r_{\mathrm{p}}^{\prime}$ by the model without considering the flux reduction and accessibility. 
440 The equation for vacant pore concentration at the inlet cross-section is 441 obtained substituting (46) into (33):

$$
h^{(0)}(T)=h_{0}^{(0)}-\sigma^{(0)}(T) .
$$

443

444

445

446

447

448

449

450

451

452

453

454

455

456

457

458

459

460

461

462

463

464

465

466

467

468

469

470

471

472

473

474

475

476

The relationship (47) reflects the fact that each particle can plug only one pore and viceversa.

For the case of a single pore size medium (37), the solution of the system (30), subject to the initial and boundary conditions (31), is given by formulae (39), (40), (42)-(47).

The plot of the solution is given in Figure 3. Initial concentration density for pores and concentration density for suspended particles at inlet cross-section are shown in Figure 3(a).

The dynamics of particle size distributions (PDF) for small and large particles is shown in Figure 3(b). Comparison between continuous and dotted lines shows that the shape of small particle concentration density is repeated with delay that is equals to $X$, which corresponds to travelling wave behaviour, (39). The continuous line in Figure $3 \mathrm{~b}$ shows that the large particle $\left(r_{\mathrm{s}}>r_{\mathrm{p}}^{\prime}\right)$ concentration density is equal to zero for any $X>0$. Figure $3 \mathrm{a}$ and $\mathrm{b}$ shows that the total vacancy concentration at the inlet cross-section decreases with time, as suggested by formula (47); the pore size distribution at $T>0$ remains delta function.

Figure 4 (line 1) shows concentration density of small particles at the core outlet for the case of constant injected concentration. The concentration equals zero until the injection of one pore volume. After particle arrival at the outlet at the moment $T=1$, the concentration at the outlet is equal to the injected concentration. The line 2 in Figure 4 shows that large particles never arrive to the core outlet.

It is important to highlight that, depending on the size, the particles in uniform pore size medium either pass or are trapped (see Equations (39) and (44)). Therefore, the deep bed filtration, where does exist an average penetration length for each size particle, does not happen in case of particulate flow in a single-size porous medium. The penetration length is zero for large particles, and is infinite for small particles.

Let us obtain equations for average concentrations for the case of particulate suspension flow in a single pore size medium.

Integration of both sides of system (38) in $r_{\mathrm{s}}$ from zero to $r_{\mathrm{p}}^{\prime}$ results in the system for average concentration of small particles

$$
\begin{aligned}
& \frac{\partial c_{1}(X, T)}{\partial T}+\frac{\partial c_{1}(X, T)}{\partial X}=0, \\
& \frac{\partial \sigma_{1}(X, T)}{\partial T}=0,
\end{aligned}
$$


where

478

479

480

482

483

484

485

486

487

488

489

490

491

492

493

494

495

496

497

498

499

500

$$
c_{1}(X, T)=\int_{0}^{r_{\mathrm{p}}^{\prime}} C\left(r_{\mathrm{s}}, X, T\right) \mathrm{d} r_{\mathrm{s}}, \quad \sigma_{1}(X, T)=\int_{0}^{r_{\mathrm{p}}^{\prime}} \Sigma\left(r_{\mathrm{s}}, X, T\right) \mathrm{d} r_{\mathrm{s}} .
$$

The solution of (48), accounting for initial and boundary conditions (31), is:

$$
c_{1}(X, T)= \begin{cases}c_{1}^{(0)}(T-X), & X<T, \\ 0, & X>T .\end{cases}
$$

The solution (49) shows that free advection (without particle capture) of small particles occurs. Thus, deep bed filtration of small particles does not happen.

Integration of both sides of the first and second equation (41) in $r_{\mathrm{s}}$ from $r_{\mathrm{p}}^{\prime}$ to infinity results in the system for average concentration of large particles $r_{\mathrm{s}}>r_{\mathrm{p}}^{\prime}$ :

$$
\begin{aligned}
& 0=-\frac{1}{\phi} \frac{\partial \sigma_{2}(X, T)}{\partial T}, \\
& \frac{\partial \sigma_{2}(X, T)}{\partial T}=\phi \int_{r_{\mathrm{p}}^{\prime}}^{\infty} \lambda\left(r_{\mathrm{s}}, r_{\mathrm{p}}^{\prime}\right) C\left(r_{\mathrm{s}}, X, T\right) \mathrm{d} r_{\mathrm{s}},
\end{aligned}
$$

where $\sigma_{2}$ is the average deposited concentration of large particles.

From first equation (50) and initial condition (31) we obtain the solution for average deposited concentration of large particles:

$$
\sigma_{2}(X, T)=0 .
$$

Substituting first equation (50) into second equation (50) we obtain:

$$
\int_{r_{\mathrm{p}}^{\prime}}^{\infty} \lambda\left(r_{\mathrm{s}}, r_{\mathrm{p}}^{\prime}\right) C\left(r_{\mathrm{s}}, X, T\right) \mathrm{d} r_{\mathrm{s}}=0 .
$$

Consequently, the average suspended particle concentration is also zero in the reservoir:

$$
\int_{r_{\mathrm{p}}^{\prime}}^{\infty} C\left(r_{\mathrm{s}}, X, T\right) \mathrm{d} r_{\mathrm{s}}=c_{2}(X, T)=0 .
$$

The solutions of (51) and (53) show that all large particles are captured at the inlet cross-section; there is no transport of large particles through porous media. 
501 In order to evaluate the effect of flux reduction and accessibility on

502

503

504

505

506

507

508

509

510

511

512

513

514

515

516

517

518 particulate suspension flow in porous media, let us ignore the flux reduction and accessibility factors in the system of governing equations (30), i.e. $\alpha=\gamma=1$. In this case, we obtain the population balance model as presented by Sharma and Yortsos (1987). Substituting $\alpha=\gamma=1$ in the first equation (30), results in:

$$
\frac{\partial C\left(r_{\mathrm{s}}, X, T\right)}{\partial T}+\frac{\partial C\left(r_{\mathrm{s}}, X, T\right)}{\partial X}=-\frac{1}{\phi} \frac{\partial \Sigma\left(r_{\mathrm{s}}, X, T\right)}{\partial T} .
$$

The second and the third equations of system (30) remain the same. So, the system of equations (30) takes the following form:

$$
\begin{aligned}
& \frac{\partial C\left(r_{\mathrm{s}}, X, T\right)}{\partial T}+\frac{\partial C\left(r_{\mathrm{s}}, X, T\right)}{\partial X}=-\frac{1}{\phi} \frac{\partial \Sigma\left(r_{\mathrm{s}}, X, T\right)}{\partial T}, \\
& \frac{\partial \Sigma\left(r_{\mathrm{s}}, X, T\right)}{\partial T}=\phi C\left(r_{\mathrm{s}}, X, T\right) \frac{\int_{0}^{r_{\mathrm{s}}} \lambda\left(r_{\mathrm{s}}, r_{\mathrm{p}}\right) r_{\mathrm{p}}^{4} H\left(r_{\mathrm{p}}, X, T\right) \mathrm{d} r_{\mathrm{p}}}{\int_{0}^{\infty} r_{\mathrm{p}}^{4} H\left(r_{\mathrm{p}}, X, T\right) \mathrm{d} r_{\mathrm{p}}}, \\
& \frac{\partial H\left(r_{\mathrm{p}}, X, T\right)}{\partial T}=-\phi \frac{r_{\mathrm{p}}^{4} H\left(r_{\mathrm{p}}, X, T\right)}{\int_{0}^{\infty} r_{\mathrm{p}}^{4} H\left(r_{\mathrm{p}}, X, T\right) \mathrm{d} r_{\mathrm{p}}} \int_{r_{\mathrm{p}}}^{\infty} \lambda\left(r_{\mathrm{s}}, r_{\mathrm{p}}\right) C\left(r_{\mathrm{s}}, X, T\right) \mathrm{d} r_{\mathrm{s}} .
\end{aligned}
$$

Let us discuss the case of a single pore size medium. In this case, $H\left(r_{\mathrm{p}}, X, T\right)$ is defined by Equation (37). The system (55) is reduced to the system (38) for small particles with $r_{\mathrm{s}}<r_{\mathrm{p}}^{\prime}$. The solution for this system is given in the Equations (39) and (40). The accessibility and flux reduction factors are equal unity for small particles, i.e. all pores are accessible, and systems (30) and (55) coincide.

For large particles with $r_{\mathrm{s}}>r_{\mathrm{p}}^{\prime}$, system (55) takes the following form:

$$
\begin{aligned}
& \frac{\partial C\left(r_{\mathrm{s}}, X, T\right)}{\partial T}+\frac{\partial C\left(r_{\mathrm{s}}, X, T\right)}{\partial X}=-\frac{1}{\phi} \frac{\partial \Sigma\left(r_{\mathrm{s}}, X, T\right)}{\partial T}, \\
& \frac{\partial \Sigma\left(r_{\mathrm{s}}, X, T\right)}{\partial T}=\lambda\left(r_{\mathrm{s}}, r_{\mathrm{p}}^{\prime}\right) \phi C\left(r_{\mathrm{s}}, X, T\right), \\
& \frac{\partial h(X, T)}{\partial T}=-\phi \int_{r_{\mathrm{p}}^{\prime}}^{\infty} \lambda\left(r_{\mathrm{s}}, r_{\mathrm{p}}^{\prime}\right) C\left(r_{\mathrm{s}}, X, T\right) \mathrm{d} r_{\mathrm{s}} .
\end{aligned}
$$

Substitution of the second equation (56) into the first one results in one equation for suspended particle population:

$$
\frac{\partial C\left(r_{\mathrm{s}}, X, T\right)}{\partial T}+\frac{\partial C\left(r_{\mathrm{s}}, X, T\right)}{\partial X}=-\lambda\left(r_{\mathrm{s}}, r_{\mathrm{p}}^{\prime}\right) C\left(r_{\mathrm{s}}, X, T\right) .
$$

The solution of the linear hyperbolic Equation (57) with initial and boundary conditions (31) for each particle population with particle size 
$r_{\mathrm{S}}$ is:

$$
C\left(r_{\mathrm{s}}, X, T\right)= \begin{cases}C^{(0)}\left(r_{\mathrm{s}}, T-X\right) \exp \left[-\lambda\left(r_{\mathrm{s}}, r_{\mathrm{p}}^{\prime}\right) X\right], & X<T, \\ 0, & X>T .\end{cases}
$$

The solution (58) shows separate deep bed filtration of each population of large particles with the particle-size-dependent filtration coefficient $532 \lambda\left(r_{\mathrm{s}}, r_{\mathrm{p}}^{\prime}\right)$.

The concentration history at the core outlet according to (58) is shown in Figure 4 by line 3. Concentration equals zero until the injection of one pore volume. At the moment $T=1$ the concentration front arrives at the core outlet, and the concentration is constant after the breakthrough. The ratio between the injected and effluent concentrations equals $\exp \left[-\lambda\left(r_{\mathrm{s}}, r_{\mathrm{p}}^{\prime}\right)\right]$, so it is always less than unity, i.e. the produced concentration density is lower than the injected concentration density.

The expression for vacancy concentration is:

$$
h(X, T)= \begin{cases}h_{0}(X)-\phi \int_{r_{\mathrm{p}}^{\prime}}^{\infty} \lambda\left(r_{\mathrm{s}}, r_{\mathrm{p}}^{\prime}\right) \exp \left[-\lambda\left(r_{\mathrm{s}}, r_{\mathrm{p}}^{\prime}\right) X\right] \times & \\ \times \int_{X}^{T} C^{(0)}\left(r_{\mathrm{s}}, T\right) \mathrm{d} T \mathrm{~d} r_{\mathrm{s}}, & X<T, \\ h_{0}(X), & X>T .\end{cases}
$$

Therefore, ignoring the fact that particles move only via larger pores, results in a separate deep bed filtration of large particle populations with different radii in a single pore size medium, while accounting for this effect results in the absence of deep bed filtration in this porous medium.

\section{6}

547

548

549

550

551

552

553

554

555

\section{Filtration in a Medium with Small Pore Size Variation}

Let us discuss porous medium with small pore size variation, i.e. pore radius varies inside the interval $\left[r_{\mathrm{p} \min }, r_{\mathrm{p} \max }\right]$, and $r_{\mathrm{p} \max }-r_{\mathrm{p} \min } \ll r_{\mathrm{p} \text { min }}$ (Figure 5(a)). Pore radius is uniformly distributed inside the interval $\left[r_{\mathrm{p} \text { min }}, r_{\mathrm{pmax}}\right]$. Injected particle radius is distributed according to any arbitrary probability distribution function, which is independent of time $f_{\mathrm{s}}^{(0)}\left(r_{\mathrm{s}}\right)$.

\subsection{ANALYTICAL SOLUTION}

Assuming a uniform pore size distribution, from (10) we obtain:

$$
H\left(r_{\mathrm{p}}, x, t\right)= \begin{cases}0, & r_{\mathrm{p}}>r_{\mathrm{p} \max } \text { or } r_{\mathrm{p}}<r_{\mathrm{p} \min }, \\ \frac{h(x, t)}{r_{\mathrm{p} \max }-r_{\mathrm{p} \min }}, & r_{\mathrm{p} \min }<r_{\mathrm{p}}<r_{\mathrm{p} \max } .\end{cases}
$$


Substitution of (60) into (14) and (19) allows obtaining expressions for flux reduction and accessibility factors for intermediate size particles $\left(r_{\mathrm{p} \min }<r_{\mathrm{s}}<r_{\mathrm{p} \max }\right)$ :

$$
\begin{aligned}
& \alpha\left(r_{\mathrm{s}}\right)=\frac{r_{\mathrm{p} \max }^{5}-r_{\mathrm{s}}^{5}}{r_{\mathrm{p} \max }^{5}-r_{\mathrm{p} \text { min }}^{5}}, \\
& \gamma\left(r_{\mathrm{s}}\right)=\frac{r_{\mathrm{p} \max }^{3}-r_{\mathrm{s}}^{3}}{r_{\mathrm{p} \max }^{3}-r_{\mathrm{p} \min }^{3}},
\end{aligned}
$$

i.e. the fractions $\alpha$ and $\gamma$ become just $r_{\mathrm{s}}$-dependent. Consequently, system (30) takes the form:

$$
\begin{aligned}
& \gamma\left(r_{\mathrm{s}}\right) \frac{\partial C\left(r_{\mathrm{s}}, X, T\right)}{\partial T}+\alpha\left(r_{\mathrm{s}}\right) \frac{\partial C\left(r_{\mathrm{s}}, X, T\right)}{\partial X}=-\frac{1}{\phi} \frac{\partial \Sigma\left(r_{\mathrm{s}}, X, T\right)}{\partial T}, \\
& \frac{\partial \Sigma\left(r_{\mathrm{s}}, X, T\right)}{\partial T}=\phi \eta\left(r_{\mathrm{s}}\right) C\left(r_{\mathrm{s}}, X, T\right), \\
& \frac{\partial H\left(r_{\mathrm{p}}, X, T\right)}{\partial T}=-\phi \frac{r_{\mathrm{p}}^{4} H\left(r_{\mathrm{p}}, X, T\right)}{\int_{0}^{\infty} r_{\mathrm{p}}^{4} H\left(r_{\mathrm{p}}, X, T\right) \mathrm{d} r_{\mathrm{p}}} \int_{r_{\mathrm{p}}}^{\infty} \lambda\left(r_{\mathrm{s}}, r_{\mathrm{p}}\right) C\left(r_{\mathrm{s}}, X, T\right) \mathrm{d} r_{\mathrm{s}},
\end{aligned}
$$

where,

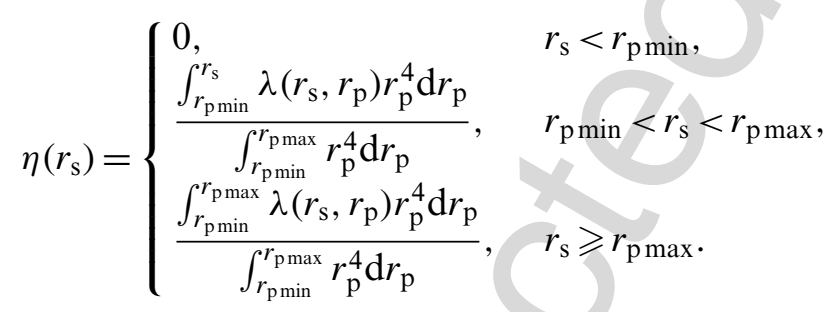

For small $\left(r_{\mathrm{s}}<r_{\mathrm{p} \min }\right)$ and large $\left(r_{\mathrm{s}}<r_{\mathrm{p} \max }\right)$ particles, system (63) coincide with systems (38) and (41), respectively. Therefore, the solution for small particles is given by formulae (39), (40) and the solution for large particles is given by (42)-(47). Small particles are transported through porous medium without being captured and all large particles are captured at the inlet cross section. Consequently, small and large particles do not perform deep bed filtration.

Figure 5(b) shows the injected particle concentration (dotted line) and the concentration density of suspended particles behind the front for $T>0$. Both concentrations coincide for small particles $\left(r_{\mathrm{s}}<r_{\mathrm{pmin}}\right)$.

On the other hand, intermediate size particles $\left(r_{\mathrm{p} \min }<r_{\mathrm{s}}<r_{\mathrm{p} \max }\right)$ perform deep bed filtration, i.e., a fraction of each particle population is captured during the transport of particles through porous media.

Let us discuss deep bed filtration of intermediate size particles.

Substitution of second Equation (63) into the first one results in:

$$
\gamma\left(r_{\mathrm{s}}\right) \frac{\partial C\left(r_{\mathrm{s}}, X, T\right)}{\partial T}+\alpha\left(r_{\mathrm{s}}\right) \frac{\partial C\left(r_{\mathrm{s}}, X, T\right)}{\partial X}=-\eta\left(r_{\mathrm{s}}\right) C\left(r_{\mathrm{s}}, X, T\right) .
$$



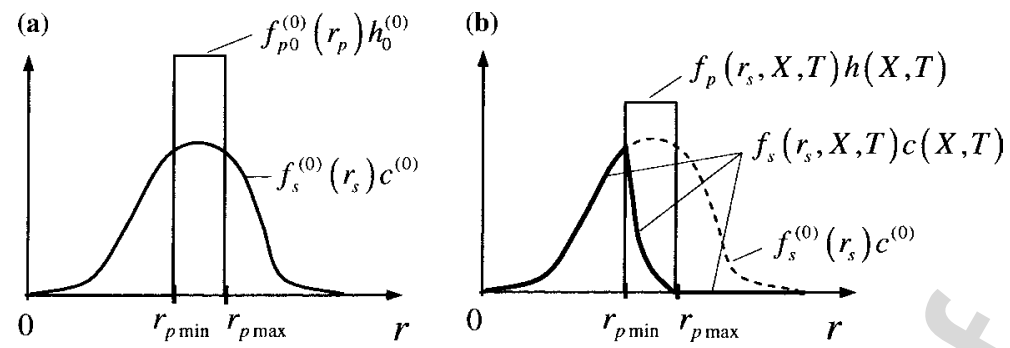

Figure 5. Distributions for suspended particles and pores in a medium with small pore size variation: (a) initial and boundary distributions for pores and suspended particles, respectively; (b) suspended particle distributions behind the concentration front for $T>0$ (solid curve) and in the injected suspension (dashed curve), and vacancy distribution.

The solution of linear hyperbolic Equation (65) is obtained by method of characteristics:

$$
C\left(r_{\mathrm{s}}, X, T\right)=\left\{\begin{array}{ll}
C^{(0)}\left(r_{\mathrm{s}}\right) \exp \left[-\frac{\eta\left(r_{\mathrm{s}}\right)}{\alpha\left(r_{\mathrm{s}}\right)} X\right], & X<\frac{\alpha\left(r_{\mathrm{s}}\right)}{\gamma\left(r_{\mathrm{s}}\right)} T \\
0, & X>\frac{\alpha\left(r_{\mathrm{s}}\right)}{\gamma\left(r_{\mathrm{s}}\right)} T
\end{array} .\right.
$$

The concentration distribution of particles with a specific size is steady state behind the concentration front, and is zero ahead of the front.

The total suspended concentration $c(X, T)$ can be calculated from (66) using formula (4).

Substituting (66) into second equation (30) and solving the resulting equation, we obtain expression for the deposited particles population:

$$
\Sigma\left(r_{\mathrm{s}}, X, T\right)= \begin{cases}\eta\left(r_{\mathrm{s}}\right) \phi\left[T-\frac{\gamma\left(r_{\mathrm{s}}\right)}{\alpha\left(r_{\mathrm{s}}\right)} X\right] C^{(0)}\left(r_{\mathrm{s}}\right) \exp \left[-\frac{\eta\left(r_{\mathrm{s}}\right)}{\alpha\left(r_{\mathrm{s}}\right)} X\right], & X<\frac{\alpha\left(r_{\mathrm{s}}\right)}{\gamma\left(r_{\mathrm{s}}\right)} T, \\ 0, & X>\frac{\alpha\left(r_{\mathrm{s}}\right)}{\gamma\left(r_{\mathrm{s}}\right)} T,\end{cases}
$$

where $\alpha\left(r_{\mathrm{s}}\right)$ and $\gamma\left(r_{\mathrm{s}}\right)$ are given by (61) and (62), respectively.

The characteristic velocity in (65) is particle-size dependent:

$$
\frac{\mathrm{d} X}{\mathrm{~d} T}=\frac{\alpha\left(r_{\mathrm{s}}\right)}{\gamma\left(r_{\mathrm{s}}\right)} .
$$

In the case where the filtration coefficient is independent of pore radius, $\lambda=\lambda\left(r_{\mathrm{s}}\right)$, from (64) we obtain:

$$
\eta\left(r_{\mathrm{s}}\right)=\lambda\left(r_{\mathrm{s}}\right)\left[1-\alpha\left(r_{\mathrm{s}}\right)\right]
$$

In the case of a bundle of parallel capillary, the dependency of the particle velocity on $r_{\mathrm{s}}$ is obtained by substitution of (61) and (62) into (68). Figure 6 shows that the larger is the particle, the larger is its velocity. The 


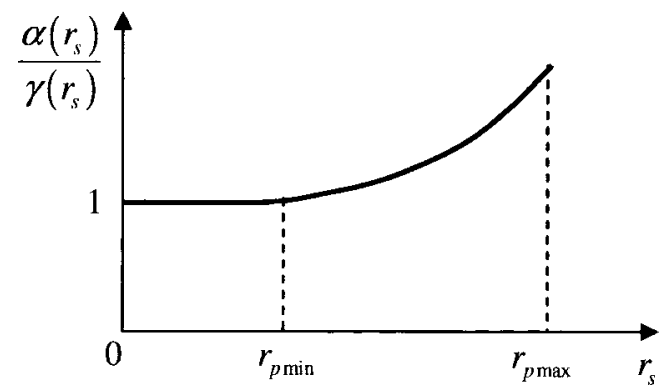

Figure 6. Particle velocity versus its radius.

603 large particles are the first to appear at the core outlet. This phenomenon 604 was observed for deep bed filtration with size exclusion of particles (Massei 605 et al., 2002) and for flow of polymer solution in a porous media (Bartelds 606 et al., 1997).

As it follows from (61), (62) and (66), for particles with $r_{\mathrm{s}}=r_{\mathrm{p} \min }(\alpha=1$ and $\gamma=1)$, there is no velocity enhancement, particles move with the velocity of carrier water.

The larger is the particle the higher is the decrement in the exponent of the solution (66). Consequently, the larger is the particle the more intensive is the particle capture rate.

When $r_{\mathrm{s}}$ tends to $r_{\mathrm{p} \max }$, the denominator in the exponent in (66) tends to zero, and the concentration tends to zero. The concentration density of intermediate size particles $C\left(r_{\mathrm{s}}, X, T\right)$ in Figure 5(b) decreases from the initial value $C^{(0)}\left(r_{\mathrm{s}}=r_{\mathrm{p} \min }\right)$ at $r_{\mathrm{s}}=r_{\mathrm{p} \min }$ to zero for $r_{\mathrm{s}}=r_{\mathrm{p} \max }$.

Substituting (60) into first equation (34) we obtain deposited concentrations at the core Inlet:

$$
\Sigma^{(0)}\left(r_{\mathrm{s}}, T\right)=\eta\left(r_{\mathrm{s}}\right) \phi C^{(0)}\left(r_{\mathrm{s}}\right) T .
$$

Here $\eta=0$ for particles with radii smaller than $r_{\text {pmin }}$ (see (64)), i.e., small particles $\left(r_{\mathrm{s}}<r_{\mathrm{p} \text { min }}\right)$ pass the core inlet without being captured. Particles with radii larger than $r_{\mathrm{pmax}}$ do not enter the rock and are deposited at the inlet cross section. From (9) follows the formula for the total deposited concentration at the core inlet:

$$
\sigma^{(0)}(T)=\int_{r_{\mathrm{p} \min }}^{\infty} \Sigma^{(0)}\left(r_{\mathrm{s}}, T\right) \mathrm{d} r_{\mathrm{s}} .
$$

Formula (33), accounting for (70) and (71), allows calculation of the total vacancy concentration at the rock inlet.

Figure 7 shows concentration profiles for different intermediate size 630 particles. The suspended concentration wave front moves with velocity $\alpha\left(r_{\mathrm{s}}\right) / \gamma\left(r_{\mathrm{s}}\right)$ 


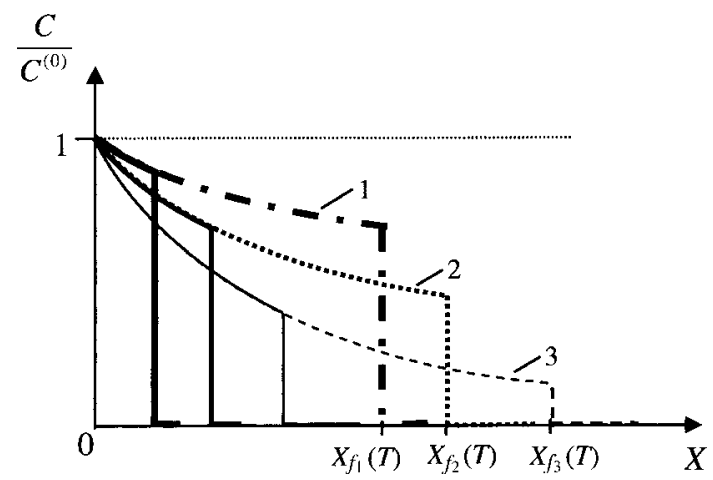

Figure 7. Concentration distribution profiles for intermediate size particles during filtration in a small pore size variation medium. Lines 1,2 and 3 correspond to different particle populations $\left(r_{\mathrm{s} 1}<r_{\mathrm{s} 2}<r_{\mathrm{s} 3}\right)$.

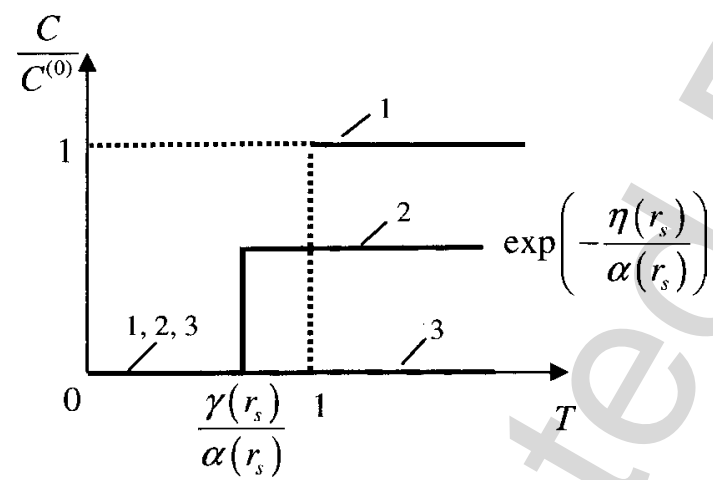

Figure 8. Particle concentration distribution histories at the core outlet, Line (1) corresponds to concentration of particles smaller than $r_{\mathrm{pmin}}$; line (2) is related to concentration of an intermediate size particles $\left(r_{\mathrm{p} \min }<r_{\mathrm{s}}<r_{\mathrm{pmax}}\right)$; line (3) corresponds to concentration of particles larger than $r_{\mathrm{pmax}}$.

The steady state profile behind the front for each particle population $C\left(r_{\mathrm{s}}, X\right)$ is given by first formula (66). Figure 7 shows that for each size particles, the profile at the moment $T_{1}$ and the section of the profile at the moment $T_{2}$ from zero to $\alpha\left(r_{\mathrm{s}}\right) / \gamma\left(r_{\mathrm{s}}\right) T_{2}$ coincide.

The larger are the particles the higher is the decrement $\eta\left(r_{\mathrm{s}}\right) / \alpha\left(r_{\mathrm{s}}\right)$ of exponent in (66), so small particles have higher relative concentration $\left(C\left(r_{\mathrm{s}}, X, T\right) / C^{(0)}\left(r_{\mathrm{s}}\right)\right)$ and their concentration profile moves slowly.

Figure 8 shows different particle size concentration history at the core outlet $(X=1)$. The larger is the particle the earlier it arrives to the outlet and the lower is its concentration afterwards.

The evolution of suspended particle concentration wave is shown in 642 Figure 9. Small particles (line 1) are not captured, porous media traps 643 intermediate size particles by pore size exclusion mechanism (lines 2 and 3), 644 and large particles do not penetrate into porous medium (line 4). 


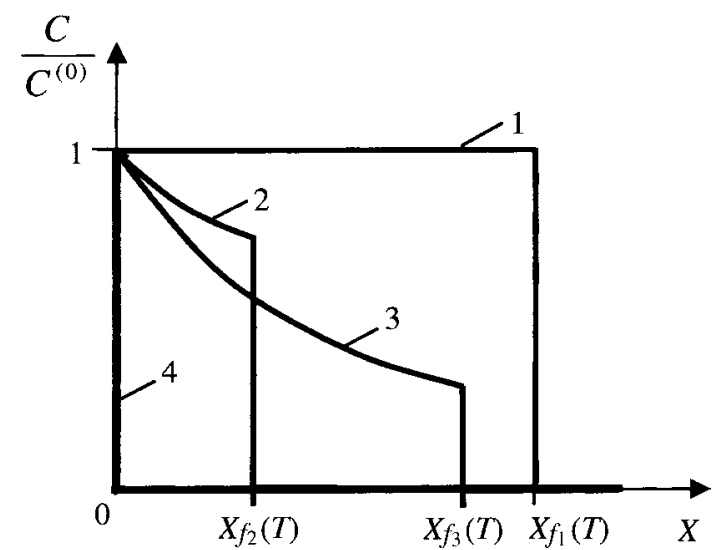

Figure 9. Concentration density profiles for different size particles. Each front moves with the velocity $\alpha\left(r_{\mathrm{s}}\right) / \gamma\left(r_{\mathrm{s}}\right)$. Line 1 corresponds to small particles $\left(r_{\mathrm{s} 1}<r_{\mathrm{pmin}}\right)$. Lines 2 and 3 are related to intermediate size particles, $r_{s 2}<r_{\mathrm{s} 3}$. Line 4 corresponds to large particles $\left(r_{\mathrm{s} 4}>r_{\mathrm{p} \max }\right)$.

In the case where the filtration coefficient is independent of pore radius, $\lambda=\lambda\left(r_{\mathrm{s}}\right)$, the explicit formulae (66) and (69) allow solving the inverse problem for determination of the filtration coefficient $\lambda\left(r_{\mathrm{s}}\right)$ from the outlet concentration data of any intermediate size particles:

$$
\lambda\left(r_{\mathrm{s}}\right)=\frac{\alpha\left(r_{\mathrm{s}}\right)}{1-\alpha\left(r_{\mathrm{s}}\right)} \ln \left(\frac{C^{(0)}\left(r_{\mathrm{s}}\right)}{C\left(r_{\mathrm{s}}, X=1\right)}\right) .
$$

\subsection{PENETRATION DEPTH}

The explicit formula (66) allows calculating average penetration depth for intermediate size particles into porous media $\left\langle X\left(r_{\mathrm{s}}, T\right)\right\rangle$ :

$$
\left\langle X\left(r_{\mathrm{s}}, T\right)\right\rangle=\frac{\int_{0}^{\frac{\alpha}{\gamma} T} X^{\prime} C\left(r_{\mathrm{s}}, X^{\prime}, T\right) \mathrm{d} X^{\prime}}{\int_{0}^{\frac{\alpha}{\gamma}} C\left(r_{\mathrm{s}}, X^{\prime}, T\right) \mathrm{d} X^{\prime}} .
$$

Particle concentration density $C\left(r_{\mathrm{s}}, X, T\right)$ is zero ahead of the propagation front $X_{\mathrm{f}}\left(r_{\mathrm{s}}, T\right)=\alpha\left(r_{\mathrm{s}}\right) / \gamma\left(r_{\mathrm{s}}\right) T$ consequently integration in (73) is performed from zero to $\left[\alpha\left(r_{\mathrm{s}}\right) / \gamma\left(r_{\mathrm{s}}\right)\right] T$. Substituting (66) into (73) and performing the integration, we obtain the formula for depth penetration dynamics:

$$
\left\langle X\left(r_{\mathrm{s}}, T\right)\right\rangle=\frac{\alpha\left(r_{\mathrm{s}}\right)}{\eta\left(r_{\mathrm{s}}\right)}\left[\frac{1-\exp \left(-\frac{\eta\left(r_{\mathrm{s}}\right)}{\gamma\left(r_{\mathrm{s}}\right)} T\right)\left(1+\frac{\eta\left(r_{\mathrm{s}}\right)}{\gamma\left(r_{\mathrm{s}}\right)} T\right)}{1-\exp \left(-\frac{\eta\left(r_{\mathrm{s}}\right)}{\gamma\left(r_{\mathrm{s}}\right)} T\right)}\right] .
$$




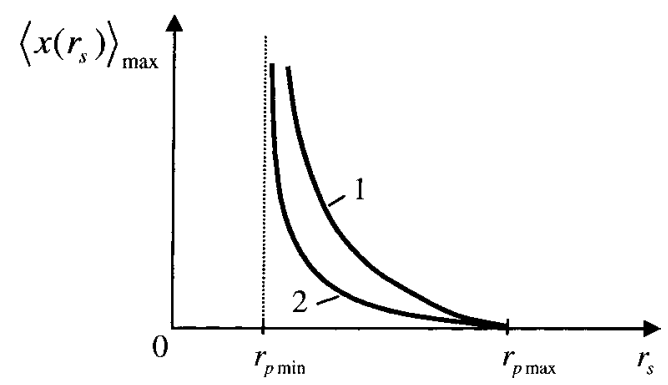

Figure 10. Effect of particle size on penetration depth $\left\langle x\left(r_{\mathrm{s}}\right)\right\rangle_{\max }$ for intermediate size particles during filtration in a small pore size variation medium.

Tending $T$ to infinity in (74), we obtain the maximum penetration depth 660 for each size particle $\left\langle X\left(r_{\mathrm{s}}\right)\right\rangle_{\max }$

$$
\left\langle X\left(r_{\mathrm{s}}\right)\right\rangle_{\max }=\frac{\alpha\left(r_{\mathrm{s}}\right)}{\eta\left(r_{\mathrm{s}}\right)} .
$$

The penetration depth does not depend on accessibility $\gamma\left(r_{\mathrm{s}}\right)$. When time tends to infinity, the suspended concentration profile given by first equation (66) is steady state and is independent of accessibility factor. Therefore, the maximum penetration depth is also accessibility-independent.

For the case where the filtration coefficient is independent of pore radius, $\lambda=\lambda\left(r_{\mathrm{s}}\right)$, substituting (69) into (75), we obtain the following maximum penetration depth:

$$
\left\langle X\left(r_{\mathrm{s}}\right)\right\rangle=\frac{\alpha}{\lambda\left(r_{\mathrm{s}}\right)(1-\alpha)} .
$$

Figure 10 shows the maximum penetration depth as a function of particle radius. Particles with radii $r_{\mathrm{s}}=r_{\mathrm{p} \max }$ do not penetrate into porous media, $\alpha$ equals zero for this case, and $\left\langle X\left(r_{\mathrm{p} \max }\right)\right\rangle_{\max }=0$. Particles with radii $r_{\mathrm{s}}=r_{\mathrm{pmin}}$ flow without being captured. In this case, $\alpha$ equals unity and $\eta\left(r_{\mathrm{s}}\right)$ tends to zero; from (75) follows that $\left\langle X\left(r_{\mathrm{p} \min }\right)\right\rangle_{\max }$ tends to infinity.

Curves 1 and 2 in Figure 10 correspond to different filtration coefficients, $\lambda_{1}<\lambda_{2}$. Particles captured less intensively penetrate deeply.

Let us analyse the effect of particle size on penetration depth. The larger is the particle, the lower is the flux reduction factor, and the smaller is the penetration depth. So, small particles penetrate deeply.

\subsection{AVERAGED CONCENTRATION MODEL}

In this section we derive an average concentration model and compare it with the classical model for deep bed filtration (Iwasaki, 1937). 
684 Let us introduce average concentrations for small, intermediate size and 685 large particles:

$$
\begin{aligned}
& c_{1}=\int_{0}^{r_{\mathrm{p} \min }} C\left(r_{\mathrm{s}}, X, T\right) \mathrm{d} r_{\mathrm{s}}, \quad c_{2}=\int_{r_{\mathrm{p} \min }}^{r_{\mathrm{p} \max }} C\left(r_{\mathrm{s}}, X, T\right) \mathrm{d} r_{\mathrm{s}}, \\
& c_{3}=\int_{r_{\mathrm{p} \max }}^{\infty} C\left(r_{\mathrm{s}}, X, T\right) \mathrm{d} r_{\mathrm{s}} .
\end{aligned}
$$

The averaged small particle concentration is obtained by integration of 689 the first equation (38) over $r_{\mathrm{s}}$ from zero to $r_{\mathrm{p} \min }$ :

$$
\frac{\partial c_{1}}{\partial T}+\frac{\partial c_{1}}{\partial X}=0
$$

Small particles move with the carrier water velocity without entrapment.

The equations for the total concentration of intermediate size particles 694 are obtained by integration of first and second equations (63) in $r_{\mathrm{s}}$ from $r_{\mathrm{p} \min }$ to $r_{\mathrm{p} \max }$ :

$$
\begin{aligned}
& \frac{\partial\left(\langle\gamma\rangle c_{2}(X, T)\right)}{\partial T}+\frac{\partial\left(\langle\alpha\rangle c_{2}(X, T)\right)}{\partial X}=-\frac{1}{\phi} \frac{\partial \sigma_{2}(X, T)}{\partial T}, \\
& \frac{\partial \sigma_{2}(X, T)}{\partial T}=\lambda \phi(1-\langle\alpha\rangle) c_{2}(X, T),
\end{aligned}
$$

where the averaged flux reduction and accessibility factors are

$$
\begin{gathered}
\langle\alpha\rangle=\frac{\int_{r_{\mathrm{p} m i n}}^{r_{\mathrm{p} \max }} \alpha\left(r_{\mathrm{s}}\right) f_{\mathrm{s}}\left(r_{\mathrm{s}}, X, T\right) \mathrm{d} r_{\mathrm{s}}}{\int_{r_{\mathrm{p} \min }}^{r_{\mathrm{p} m}} f_{\mathrm{s}}\left(r_{\mathrm{s}}, X, T\right) \mathrm{d} r_{\mathrm{s}}}, \\
\langle\gamma\rangle=\frac{\int_{r_{\mathrm{p} \min }}^{r_{\mathrm{p} \max }} \gamma\left(r_{\mathrm{s}}\right) f_{\mathrm{s}}\left(r_{\mathrm{s}}, X, T\right) \mathrm{d} r_{\mathrm{s}}}{\int_{r_{\mathrm{p} \min }}^{r_{\mathrm{p} \max }} f_{\mathrm{s}}\left(r_{\mathrm{s}}, X, T\right) \mathrm{d} r_{\mathrm{s}}} .
\end{gathered}
$$

699

The averaged flux reduction and accessibility factors change during particle retention. The particle retention is described by the deposited concentration $\sigma_{2}$. Thus, we close the system (79) introducing constitutive relations

$$
\langle\alpha\rangle=\langle\alpha\rangle\left(\sigma_{2}\right) \text { and }\langle\gamma\rangle=\langle\gamma\rangle\left(\sigma_{2}\right) .
$$

If compared with the classical deep bed filtration model (1), the model (79) for intermediate size particles contains flux reduction term (80) and accessibility factor (81) in the population balance equation. The capture rate expression in (79) contains the factor $(1-\langle\alpha\rangle)$ showing that the capture rate should be proportional not to the overall flow velocity $U$ as 
709 it is assumed in (1), but to the fraction of the flow velocity via small pores

$710(1-\langle\alpha\rangle) U$.

711 The equations for large particle concentrations $c_{3}$ and $\sigma_{3}$ are obtained 712 by integration of equations (41) in $r_{\mathrm{s}}$ from $r_{\mathrm{p} \max }$ to infinity. The averaged 713 equations are the same as Equations (50) for large particles.

\section{7. Deep Bed Filtration in a Simple Geometry Medium}

715 Let us derive the population balance model for deep bed filtration in a 716 simplified geometry porous medium, which is a bundle of parallel capillary alternated by mixing chambers (Figure 2).

Particles are assumed to be deposited on sieves; $\sigma^{\prime}(x, t)$ is deposited particle concentration per unit of a sieve area, the vacancy concentration $h^{\prime}(x, t)$ is also determined per unit of a sieve area:

$$
\sigma^{\prime}=\sigma l, \quad h^{\prime}=h l .
$$

The number of particles with radius from the interval $\left[r_{\mathrm{s}}, r_{\mathrm{s}}+\mathrm{d} r_{\mathrm{s}}\right]$ captured in pores with radius from the interval $\left[r_{\mathrm{p}}, r_{\mathrm{p}}+\mathrm{d} r_{\mathrm{p}}\right]$ per unit of time is equal to the number of particles with radius from the interval $\left[r_{\mathrm{s}}, r_{\mathrm{s}}+\right.$ $\mathrm{d} r_{\mathrm{s}}$ ] arriving to the sieve multiplied by water flux via pores with radius the interval $\left[r_{\mathrm{p}}, r_{\mathrm{p}}+\mathrm{d} r_{\mathrm{p}}\right]$ :

$$
\begin{aligned}
& \frac{\partial \sigma^{\prime}(x, t) \frac{f_{T}}{\partial t}\left(r_{\mathrm{s}}, r_{\mathrm{p}}, x, t\right)}{\partial t} \mathrm{~d} r_{\mathrm{s}} \mathrm{d} r_{\mathrm{p}} \\
& \quad=c(x, t) f_{\mathrm{s}}\left(r_{\mathrm{s}}, x, t\right) U \frac{r_{\mathrm{p}}^{4} f_{\mathrm{p}}\left(r_{\mathrm{p}}, x, t\right)}{\int_{0}^{\infty} r_{\mathrm{p}}^{4} f_{\mathrm{p}}\left(r_{\mathrm{p}}, x, t\right) \mathrm{d} r_{\mathrm{p}}} \mathrm{d} r_{\mathrm{s}} \mathrm{d} r_{\mathrm{p}} .
\end{aligned}
$$

Integrating both parts of (84) in $r_{\mathrm{p}}$ from zero to $r_{\mathrm{s}}$ and accounting for (6) result in the expression for the total capture rate of particles with radius $731 r_{\mathrm{s}}$ in a single sieve:

$$
\frac{\partial}{\partial t}\left(\sigma^{\prime}(x, t) f_{T}\left(r_{\mathrm{s}}, x, t\right)\right)=c(x, t) f_{\mathrm{s}}\left(r_{\mathrm{s}}, x, t\right) \frac{U \int_{0}^{r_{\mathrm{s}}} r_{\mathrm{p}}^{4} f_{\mathrm{p}}\left(r_{\mathrm{p}}, x, t\right) \mathrm{d} r_{\mathrm{p}}}{\int_{0}^{\infty} r_{\mathrm{p}}^{4} f_{\mathrm{p}}\left(r_{\mathrm{p}}, x, t\right) \mathrm{d} r_{\mathrm{p}}} .
$$

Changing areal deposited concentration in a sieve per volumetric deposited concentration (see (83)) and substituting formulae (3), (8) and (10) in (85), we obtain:

$$
\frac{\partial \Sigma\left(r_{\mathrm{s}}, x, t\right)}{\partial t}=\frac{1}{l} C\left(r_{\mathrm{s}}, x, t\right) \frac{U \int_{0}^{r_{\mathrm{s}}} r_{\mathrm{p}}^{4} H\left(r_{\mathrm{p}}, x, t\right) \mathrm{d} r_{\mathrm{p}}}{\int_{0}^{\infty} r_{\mathrm{p}}^{4} H\left(r_{\mathrm{p}}, x, t\right) \mathrm{d} r_{\mathrm{p}}} .
$$
filtration coefficient $\left(\lambda^{\prime}\right)$ equals the inverse to the distance between the sieves. 
740 It is assumed that in each sieve one particle can plug only one pore, and vice versa. So, formula (12) can be applied to concentrations in each sieve:

$$
h^{\prime}(x, t) f_{\mathrm{p}}\left(r_{\mathrm{p}}, x, t\right)=h_{0}^{\prime}(x) f_{\mathrm{p} 0}\left(r_{\mathrm{p}}, x\right)-\int_{r_{\mathrm{p}}}^{\infty} \sigma^{\prime}(x, t) \underline{f_{T}}\left(r_{\mathrm{s}}, r_{\mathrm{p}}, x, t\right) \mathrm{d} r_{\mathrm{s}} .
$$

Differentiating (87) with respect to $t$ and substituting (84) in the resulting equation, we obtain the pore plugging kinetics:

$$
\begin{aligned}
\frac{\partial}{\partial t}\left(h^{\prime}(x, t) f_{\mathrm{p}}\left(r_{\mathrm{p}}, x, t\right)\right)= & -\frac{f_{\mathrm{p}}\left(r_{\mathrm{p}}, x, t\right) r_{\mathrm{p}}^{4}}{\int_{0}^{\infty} f_{\mathrm{p}}\left(r_{\mathrm{p}}, x, t\right) r_{\mathrm{p}}^{4} \mathrm{~d} r_{\mathrm{p}}} U c(x, t) \times \\
& \times \int_{r_{\mathrm{p}}}^{\infty} f_{\mathrm{s}}\left(r_{\mathrm{s}}, x, t\right) \mathrm{d} r_{\mathrm{s}} .
\end{aligned}
$$

Changing areal vacancy concentration in a sieve per volumetric vacancy concentration (see (83)) and substituting formulae (3), (8) and (10) in the resulting equation, we obtain:

$$
\frac{\partial H\left(r_{\mathrm{p}}, x, t\right)}{\partial t}=-\frac{1}{l} \frac{H\left(r_{\mathrm{p}}, x, t\right) r_{\mathrm{p}}^{4}}{\int_{0}^{\infty} H\left(r_{\mathrm{p}}, x, t\right) r_{\mathrm{p}}^{4} \mathrm{~d} r_{\mathrm{p}}} U \int_{r_{\mathrm{p}}}^{\infty} C\left(r_{\mathrm{s}}, x, t\right) \mathrm{d} r_{\mathrm{s}} .
$$

The system of governing equations for deep bed filtration ((89) and (86)) in a bundle of parallel capillary alternated by mixing chambers coincide with the system (28) proposed for a general case of pore space geometry.

The dimensional filtration coefficient for deep bed filtration in a bundle of parallel capillary alternated by mixing chambers equals the inverse to the distance between the sieves, i.e. is constant. It coincides with the pore plugging kinetics suggested by Sharma and Yortsos (1987a) where $l$ is considered to be equal to the pore length.

\section{8. Conclusions}

760 Derivation of the stochastic deep bed filtration model for size exclusion mechanism accounting for particle flux reduction and pore accessibility effects, and analytical solutions obtained allow for the following conclusions:

1. Absence of particles in the pores that are smaller than the particles, results in reduction of the particle carrying water flux if compared with the overall water flux. It also means that only a fraction of the pore space is accessible for particles. The flux reduction term appears in the advection flux in the population balance equation; the accessibility factor appears in the accumulation term. 
806

807 808

2. The analytical solution for flow in a single pore size $r_{\mathrm{p}}^{\prime}$ medium shows that capture-free advection of small particles $\left(r_{\mathrm{s}}<r_{\mathrm{p}}^{\prime}\right)$ takes place, and large particles $\left(r_{\mathrm{s}}>r_{\mathrm{p}}^{\prime}\right)$ do not penetrate into the porous medium. Consequently, there is no deep bed filtration in a uniform pore size medium. Ignoring flux reduction and accessibility effects results in a separate deep bed filtration of large different size particles.

3. The analytical solution for flow in a porous media with small pore size variation shows that the particles larger than all pores do not move and that the particles smaller than pores move through the media without capture.

The intermediate size particles perform deep bed filtration. Populations with different size particles filtrate independently; the filtration coefficient and the flux reduction and accessibility factors for each population are particle-size-dependent.

4. The larger is the intermediate size particle, the lower is its penetration depth during deep bed filtration in the rock with small pore size variation.

5. The average concentration models can be derived for flow in porous media with small pore size variation for small particles, for intermediate size particles and for large particles separately.

The averaged model for intermediate size particles differs from the traditional deep bed filtration model by the flux reduction and accessibility factors $(\langle\alpha\rangle$ and $\langle\gamma\rangle$, respectively), that appear in the particle balance equation. Also, the capture rate in the averaged model is proportional to the water flux via inaccessible pores, while in the traditional model it is proportional to the overall water flux.

\section{Acknowledgements}

Authors are mostly grateful to Petrobras colleagues A. G. Siqueira, A. L. de Souza and F. Shecaira for fruitful and motivating discussions. Many thanks are due to Prof. A. Shapiro (Denmark Technical University) for the useful suggestions and improvement of the text.

The detailed discussions with Prof. Yannis Yortsos (USC) are highly acknowledged.

Especial gratitude is due to Themis Carageorgos and Poliana Deolindo (North Fluminense State University UENF, Brazil) for permanent support and encouragement.

\section{References}

Bartelds, G. A., Bruining, J. and Molenaar, J.: 1997, The modeling of velocity enhancement in polymer flooding, Transport Porous Media 26, 75-88. 
Bedrikovetsky, P., Marchesin, D., Checaira, F., Serra, A. L. and Rezende, E.: 2001, Characterization of deep bed filtration system from laboratory pressure drop measurements, J. Petr. Sci. Eng. 64(3), 167-177.

Bedrikovetsky, P., Tran, P., Van den Brock, W. M. G., Marchesin, D., Rezende, E., Siqueira, A., Serra, A. L. and Shecaira, F.: 2003, Damage characterization of deep bed filtration from pressure measurements, J. Soc. Petr. Eng: Prod. Facilities, 3, 119-128.

Dawson, R. and Lantz R. B.: 1972, Inaccessible pore volume in polymer flooding, Soc. Petr. Eng. J. 448-452, October.

Elimelech, G., Gregory, J., Jia, X. and Williams, R. A.: 1995, Particle Deposition and Aggregation, Butterworth-Heinemann, USA.

Herzig, J. P., Leclerc, D. M. and Goff, P. le.: 1970, Flow of suspensions through porous media - application to deep filtration, Ind. Eng. Chem. 62(5), 8-35.

Iwasaki, T.: 1937, Some notes on sand filtration, J. Am. Water Works Ass. 1591-1602.

Khatib, Z. I.: 1994, Prediction of Formation Damage Due to Suspended Solids: Modeling Approach of Filter Cake Buildup in Injectors, 1995, SPE paper 28488 presented at SPE 66t9th Annual Technical Conference and Exhibition held in New Orleans, LA, USA, 2528 September

Khilar, K. and Fogler, S.: 1998, Migration of Fines in Porous Media, Kluwer Academic Publishers, Dordrecht/London/Boston.

Landau, L. D. and Lifshitz, E. M.: 1980, Statistical Physics (Course on Theoretical Physics, V.5), 3rd edition, Pergamon Press, Oxford, UK.

Landau, L. D. and Lifshitz, E. M.: 1987, Fluid Mechanics(Course on Theoretical Physics, V.6), 2nd edition, Pergamon Press, Oxford. UK.

Massei, N., Lacroix, M., Wang, H. Q. and Dupont, J.: 2002, Transport of particulate material and dissolved tracer in a highly permeable porous medium: comparison of the transfer parameters, J. Contam. Hydro. 57, 21-39.

Oort, E. V., Velzen, J. F. G. V. and Leerlooijer, K.: 1993, Impairment by Suspended Solids Invasion: Testing and Prediction, SPE paper 23822.

Pang, S. and Sharma, M. M.: 1994, A Model for Predicting Injectivity Decline in Water Injection Wells. SPE paper 28489, 275-284.

Payatakes, A. C., Tien, C. and Turian, R. M.: 1973, A new model for granular porous media. I. model formulation, AIChE J., 19(1), 58-76.

Payatakes, A. S., Rajagopalan, R. and Tien, C.: 1974, Application of porous medium models to the study of deep bed filtration, The Canadian J. Chem. Eng. 52.

Rege, S. D. and Fogler, H. S.: 1988, A network model for deep bed filtration of solid particles and emulsion drops, AIChE J. 34(11), 1761-1772.

Sahimi, M. and Imdakm, A. O.: 1991, Hydrodynamics of particulate motion in porous media. Phys. Rev. Letters 66(9), 1169-1172.

Seljakov, V. I. and Kadet, V. V.: 1996, Percolation Models in Porous Media, Kluwer Academic, Dordrecht-NY-London.

Sharma, M. M. and Yortsos, Y. C.: 1987a, Transport of particulate suspensions in porous media: model formulation, AIChe J. 33(10), 1636.

Sharma, M. M. and Yortsos, Y. C.: 1987b, A network model for deep bed filtration processes, AIChE J. 33(10), 1644-1653.

Sharma, M. M. and Yortsos, Y. C.: 1987c, Fines migration in porous media, AlChE J. 33(10), 1654-1662.

Siqueira, A. G., Bonet, E. and Shecaira, F. S.: 2003, Network modelling for transport of water with particles in porous media. SPE paper 18257 presented at the SPE Latin American and Caribbean Petroleum Engineering Conference held in Port-of-Spain, Trinidad and Tobago. 
859 Tikhonov, A. N. and Samarskii, A. A.: 1990, Equations of Mathematical Physics, Dover, $860 \quad$ New York.

861 Veerapen, J. P., Nicot, B. and Chauveteau, G. A.: 2001, In-Depth Permeability Damage by 862 Particle Deposition at High Flow Rates, SPE paper 68962 presented at presented at the 863 SPE European Formation Damage Conference to be held in The Hague, The Netherlands 864 21-22 May 2001.

865 Wennberg, K. E. and Sharma, M. M.: 1997, Determination of the filtration coefficient and 866 the transition time for water injection, SPE 38181, 353-364. 